\title{
SUR L'ÉTUDE \\ DU LANGAGE ENFANTIN
}

\author{
par Marcel Cohen
}

\section{INDICATIONS PRÉALABLES}

L'observation du langage enfantin ne peut être pleinement valable que si on tient compte de toute la complication de la matière et des difficultés de la tâche. Il ne faut pas perdre de vue un seul moment qu'on se trouve constamment devant un champ qui va du musculaire à l'intellectuel, et qu'on est toujours dans la complexité du social.

A vrai dire, cette étude devrait être le fait de professionnels spécialisés, au moins au cours de certaines parties de leur carrière. Une science bien organisée devrait comprendre des chaires de langage enfantin, dont les titulaires organiseraient les recherches en utilisant des collaborations partielles, en particulier en donnant l'enseignement linguistique nécessaire à la partie du personnel des pouponnières et des crèches qui manifesterait. un goût suffisant pour ce travail. Lcs quelques travaux réalisés jusqu'ici, sur le domaine de quelques langues européennes, ont été faits en marge de multiples autres tâches; tous sont partiels; même les meilleurs présentent dans le détail des manques bien naturels, les linguistes les plus exercés n'ayant pas toujours en tête les questions biologiques et sociologiques, de même que les psychologues manquent de certaines connaissances linguistiques, tous ayant défriché en pionniers et dans de mauvaises conditions de réalisation.

L'auteur du présent mémoire, qui n'a pas disposé de meilleures conditions, ne prétend pas tracer en quelques pages les instructions générales, avec des cadres admis de tout le monde, qu'il faut prévoir pour l'avenir.

En se fondant sur quelques observations personnelles et sur la lecture d'ouvrages récents qui eux-mêmes pour leur part résument la majeure partie de la littérature antérieure, il vise à attirer l'attention sur des faits mal mis en lumière jusqu'ici et à ñréparer ainsi la voie aux études méthodiques qu'il est hautement désirable de voir se développer. 
Ici, une mise en garde à l'usage des futurs travailleurs.

Faute d'un avancement suffisant de la recherche, d'une diffusion suffisante de certaines connaissances acquises et quelquefois d'une élémentaire prudence de certains spécialistes en ce qui concerne des spécialités qui ne sont pas la leur, des notions imaginaires mais invétérées peuvent encore se trouver sous la plume ou dans la bouche de savants dont par ailleurs les enseignements sont d'une incontestable valeur.

Ce qui est le plus répandu et le plus spectaculaire en cette matière est la conviction souvent exprimée qu'il y a priorité pour les mots maman et papa, parce que ces mots sont liés à la naturelle affectivité de l'enfant, et parce qu'ils comprennent essentiellement des consonnes labiales, ceci en liaison avec l'usage précoce des lèvres. Or, chez la majorité sinon la totalité des enfants qui ont été bien observés ces mots n'ont pas été les premiers; de plus, dans de nombreuses langues des désignations des parents ne comportent pas de labiales (ainsi russe otiets " père n); enfin - ne devrait-on pas dire surtout? - les lèvres ne jouent qu'un rôle passif dans la tétée, la succion étant opérée par le ventousage au moyen de l'arrière de la langue.

- Il sera sûrement long d'extirper la légende dans le public : la conviction enracinée que maman et papa sont les premiers mots fait habituellement négliger l'observation de ce que l'enfant exprime avant l'établissement desdits mots, d'autant plus qu'il s'agit généralement d'éléments mal compréhensibles, soit créations enfantines soit termes adultes très altérés; on ne demande donc pas à quel âge l'enfant a dit son premier not, mais à quel âge il a dit papa et maman.

Pour les chercheurs, ils se garderont mieux des notions soi-disant acquises s'ils se disent qu'en général tout est à reprendre, et que d'ailleurs un grand nombre des questions qui se posent sont actuellement insolubles. Disons bien haut qu'on ne pourra pas avancer au-delà des premiers pas sans avoir d'une part des observations linguistiques méthodiques en nombre suffisant dans le plus grand nombre de langues possible, sans d'autre part que de nouveaux pas en avant soient faits par la psychologie scientifique, sans compter la sociologie.

Autre chose. On admet généralement que la linguistique est seulement une science d'observation, quelquefois d'observation instrumentale, pour la phonétique. En réalité, par certaines mesures concernant l'enseignement de la langue maternelle ou celui d'une langue plus ou moins imposée à des populations d'autre langue, des expériences sont pratiquement instituées en matière de langage. Ce sont des questions qui mériteraient des études nombreuses. Pour ce qui est de la période d'acquisition du langage maternel ou des langages familiaux, beaucoup de possibilités sont ouvertes à l'expérience. Certains linguistes ont usé de celle qui consiste à instituer un bilinguisme voulu dans la petite société familiale. Il ne peut pas y avoir d'inconvénients sérieux à de 
telles expériences : en mettant les choses au pire, certains retards de quelques mois - qui pourraient être compensés par des avantages imprévus - n'auraient rien de tragique.

Que les conditions soient expérimentales ou non, les faits sont recueillis par l'observation. Celle-ci s'est faite jusqu'à présent presque toujours à l'audition (et à la vue en tant que celle-ci est en question), avec des notations écrites en écriture phonétique dans les meilleurs cas. L'usage des instruments enregistrants devrait être maintenant institué en grand. Ceci suppose naturellement les commodités et les crédits nécessaires.

\section{DocUmentatron RÉCENTE}

Werner F. Leopold, Speech development of a bilingual child, a linguist's record, Northwestern University. Evanston-Chicago. Volume $\mathbf{L}$ Vocabulary growth in the first tspo years, 1939. Volume II. Sound learning in the first two years, 1947. Volume III. Grammar and general problems in the first tswo years, 1949. Volume IV. Diary from age 2, 1949. Ouvrage très riche, avec bibliographie. Le journal du volume IV pousse certaines observations jusqu'à l'adolescence. In s'agit en réalité de deux filles nées le 3 juillet 1930 et le 18 juillet 1936 à qui la mère parlait anglais en milieu américain de langue anglaise, tandis que le père leur parlait allemand, et dont l'aînée a fait des voyages en Allemagne.

Comptes rendus de Marcel Cohen dans le Bulletin de la Sociéte de Linguistique [cité ensuite B. S. L.]. T. 41, 1941, p. 9-11; t. 46, 1950, p. 25-27; t. 47, 1951 [cité Leopold].

Antoine Grégotre, L'Apprentissage du langage des deux premières années, Liége-Paris, 1937. T. II. La Troisième Année et les années suipantes, Liége, 1947. Livre très riche. Observation des deux fils de l'auteur, nés le 7 novembre 1903 et le 15 septembre 1905.

Comptes rendus de W. F. Leopold dans Language, 1948, et de Marcel Cohen dans B. S. L., t. 38, 1937, p. 6-8, et 46, 1950, p. 23-25 [cité Grégoire].

Karel Ohaseng, Etude phonétique sur le langage de l'enfant (en tchèque avec résumé en français). Prague, Université Charles, 1948. Observation d'un garçon tchèque né en 1942. L'attention est portée en particulier sur l'intonation. Vocabulaire de 1.920 mots à la fin de la $4^{\mathrm{e}}$ année. Petite bibliographie.

- Sur le développement du langage de l'enfant (en tchèque), Prague, 1948.

François Rostand, Grammaire et affectivité, Paris, 1951. Certaines observations personnelles et résumés d'études antérieures, avec une bibliographie poussée en partie jusqu'à 1950; contient des références et des citations psychanalytiques à interprétations sexuelles qui sont à rejeter.

Compte rendu par Marcel Cohen, B. S. L., t. 47 [cité Rostand]. 
La bibliographie (groupée dans Bibliography of child language par Werner F. Leopold, Evanston 1952, parue pendant l'impression du présent article) est à compléter au moyen des périodiques psychologiques et pédagogiques.

Certains des plus récents manuels de psychologie sont cités ici :

Manual of Child psychology edited by Leonard Carmichaer, New-York Londres, 1946. Chapitre 10 : Language development in children, p. 476-581 (bibliographie à partir de p. 568), par Dorothea Mc Carthy, qui a publié d'autre part des observations sur le langage enfantin. (Traduction française, t. II, 1952).

Paul Guxlaume, Manuel de Psychologie, Paris, 1950, § 165, p. 254-256. L'acquisition individuelle du langage avec utilisation d'observations personnelles de l'auteur, dont les articles sur le langage enfantin ont leur place par ailleurs dans les bibliographies.

Marcel Bergeron, Psychologie du premier áge, Paris, 1951. Le Langage, p. 77-85. Renseignements de seconde main; généralisant indûment certains faits (notamment pour l'âge de l'interrogation).

Pour la théorie, il convient de citer spécialement certaines contributions.

A. Sécheнaye, Essai sur la structure logique de la phrase, Paris, 1926 [non cité par Leopold, utilisé par Rostand], dont les observations et les interprétations (mot-phrase ou monorème $=$ prédicat de la situation) sont la source de développements d'auteurs postérieurs.

Mrs. E. Norman, Some psychological features of babble in children, dans Proceedings of the second international Congress of phonetic sciences, Londres, 1935 (Cambridge, 1936), no 36, p. 155-158. [Omis par Leopold]. Intéressant pour le gazouillis pendant la période où le langage s'institue.

Th. Sxmon, Étude psychologique de l'enfant, série d'articles dans le Bulletin de la Societé Alfred Binet, 1948-1950. Voir en particulier dans le $n^{0} 383$ de juin-juillet 1948, sur l'acquisition du langage [non cité par Leopold et Rostand].

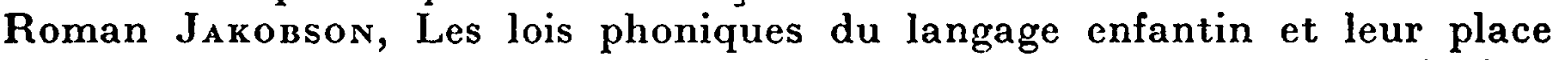
dans la phonologie générale, dans N. S. Troubetskox, Principes de phonologie, traduction J. Cantineau, Paris, 1949, p. 367-379. Communication au Congrès des Linguistes de Bruxelles, septembre 1939, qui ne s'est tenu qu'en partie et dont les Actes n'ont pas paru. Reproduit l'cssentiel des idées exposées par l'auteur dans une brochure en allemand, Upsal, 1941, actuellement épuisée [citée et utilisée par Leopold].

Marguerite Durand, Le Langage enfantin, conférence de l'Institut de Linguistique de l'Université de Paris, à paraître en 1953.

Les indications ci-dessous, qui ne sont pas données comme complètes, concernent des langues diverses en dehors de l'Europe.

En domaine sémitique, des mots du langage enfantin, plus ou moins employés par les adultes, sont cités à l'occasion dans les dictionnaires et les ouvrages descriptifs. 
Pour l'arabe moderne : Marcel Conen, Le Parler arabe des juifs d'Alger, Paris, 1912 (à l'index : hypocoristiques); Enno Lrtrmann, Kinderlieder und Kindersprache im heutigen Aegypten, dans Mélanges Maspero, III, 1935 (Mémoires de l'Institut français du Caire, t. LXVIII), p. 231-240.

Le document le plus important est Carl Reinhard, Ein arabischer Dialekt gesprochen im Omān und Zanzibar, Stuttgart-Berlin, 1894, $\S 229$, p. 125-126 : Kindersprache. Une liste est donnée en notation phonétique (par exemple lōlōh " dodo"). Il est dit que certaines consonnes de la langue normale sont remplacées par d'autres; ainsi yas pour ras " tête". L'âge exact n'est pas indiqué.

Un professeur égyptien doit faire un ouvrage sur l'acquisition par les enfants de l'arabe, puis d'une langue étrangère à l'âge scolaire.

Pour une langue bantoue de la région du lac Tanganika, on a : O. F. RAUM, Chaga Childhood, publication de International Institute of African Languages and Cultures, Oxford, 1940 : The development of speech as instrumental behaviour, p. 146-154. Quelques observations directes, montrant des faits homologues à ceux constatés pour les langues européennes.

Pour le chinois : Yuen Ren Chao, The Cantian idinlect, An analysis of the Chinese spoken by a twenty-eight-months-old child, dans Semitic and oriental studies presented to William Popper, University of California Publications in Semitic philology, vol. XI, 1951, p. 27-44. Diverses simplifications, notamment pour l'emploi des tons, ce qui multiplie les homonymies. Vocabulaire actif de 320 mots environ.

\section{Contributions antérieures de l'auteur.}

Marcel Conen, Sur les langages successifs de l'enfant. Mélanges Vendryes, 1925, p. 109-127 [cité Langages successifs].

- A propos de la 3 e personne du féminin du pluriel en français. B. S. L., t. XXVII, 1927, p. 201-208.

- Les dernières persistances du langage enfantin. Journal de Psychologie, t. 30, 1933, p. 390-399.

Le langage (Structure et évolution); Paris, 1950. Surtout p. 13-14.

- Langage enfantin. Communication à la Société de Linguistique préliminaire au présent ouvrage (avant la résolution de ne pas appliquer au langage enfantin le vocabulaire du langage adulte). Discussion par divers membres de la Société. B. S. L., t. 47, 1951, p. $\times x \times v-x \times x v i$.

\section{SUJETS OBSERVÉS}

$\left.1^{\circ}\right)$ Enfants et petits enfants de l'auteur :

Francis Cohen, né le 12 mai 1914 [cité Fr.].

Laurence Cohen, née le 26 mars 1920 [citée L.].

Christiane Cohen, née le 26 avril 1921 [citée Chr.]. 
Jérôme Lentin, né le 12 juin 1949, fils de L. et d'André Lentin [cité J.] Jean-Louis Cohen, né le 20 juillet 1949, fils de Fr. et de Marie-Élisa Cohen [cité J. L.].

20) Françoise Rouquès, née le 9 novembre 1946; observations de son père Pierre Rouquès (une fiche de Marcel Cohen).

Pour les observations des cinq premiers sujets, les âges sont indiqués par les mois et un compte de jours exact à deux ou trois unités près ou par une fraction approximative. Pour le sixième sujet le mois est indiqué.

\section{NOTATION PHONÉTIQUE}

Pour éviter toute confusion et pouvoir tenir compte des détails d'articulation, il faut employer de préférence non une orthographe nationale mais une écriture phonétique.

Si on emploie une orthographe nationale, en particulier l'orthographe française, il faut la standardiser.

Le tableau abrégé suivant donne à la fois un type d'écriture phonétique qui est employé dans le présent travail et la correspondance en orthographe française standardisée; quelques signes complémentaires sont indiqués pour ce que l'alphabet français ne peut pas noter.

\section{Voyelles.}

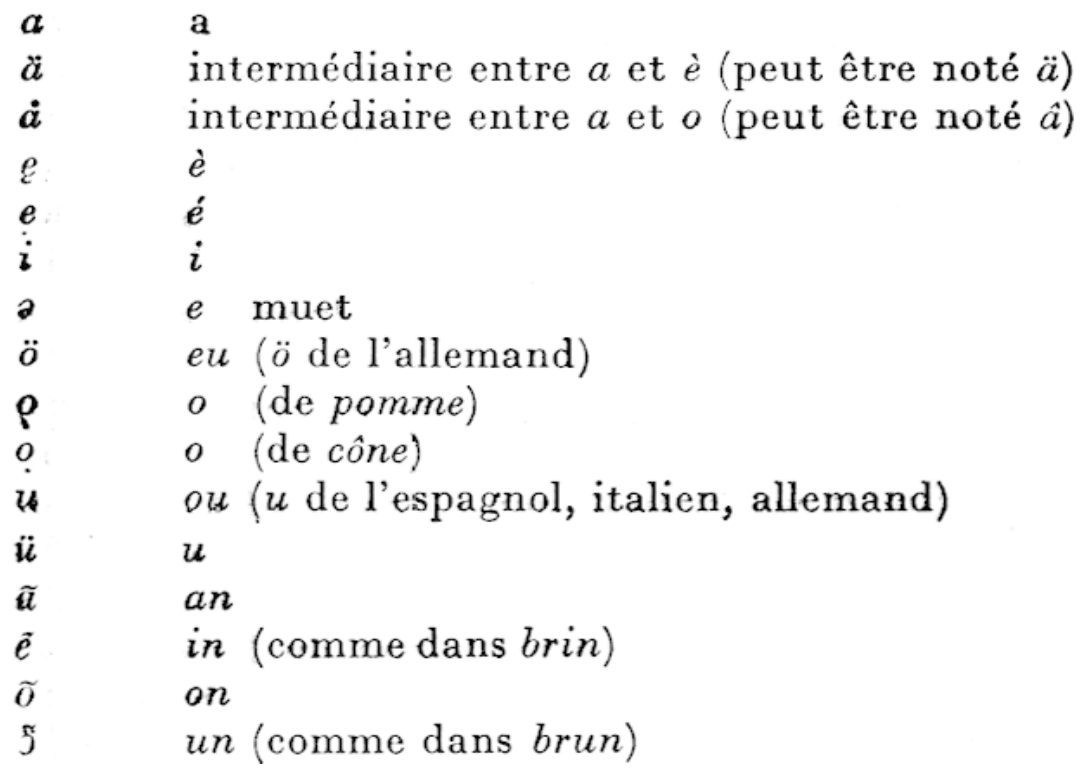

Note. - Beaucoup de Français ne savent pas prononcer $\delta$ et prononcent brun comme brin.

Semi-soyelles.

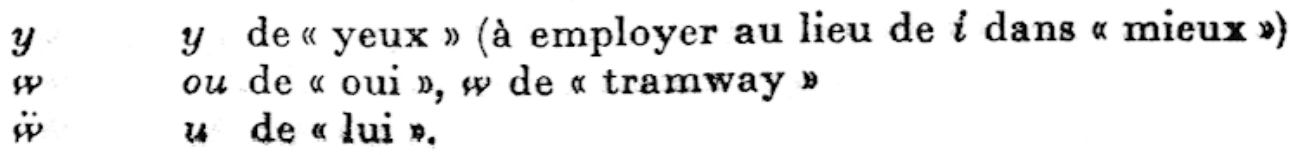




\section{Consonnes autres que les liquides et les nasales}

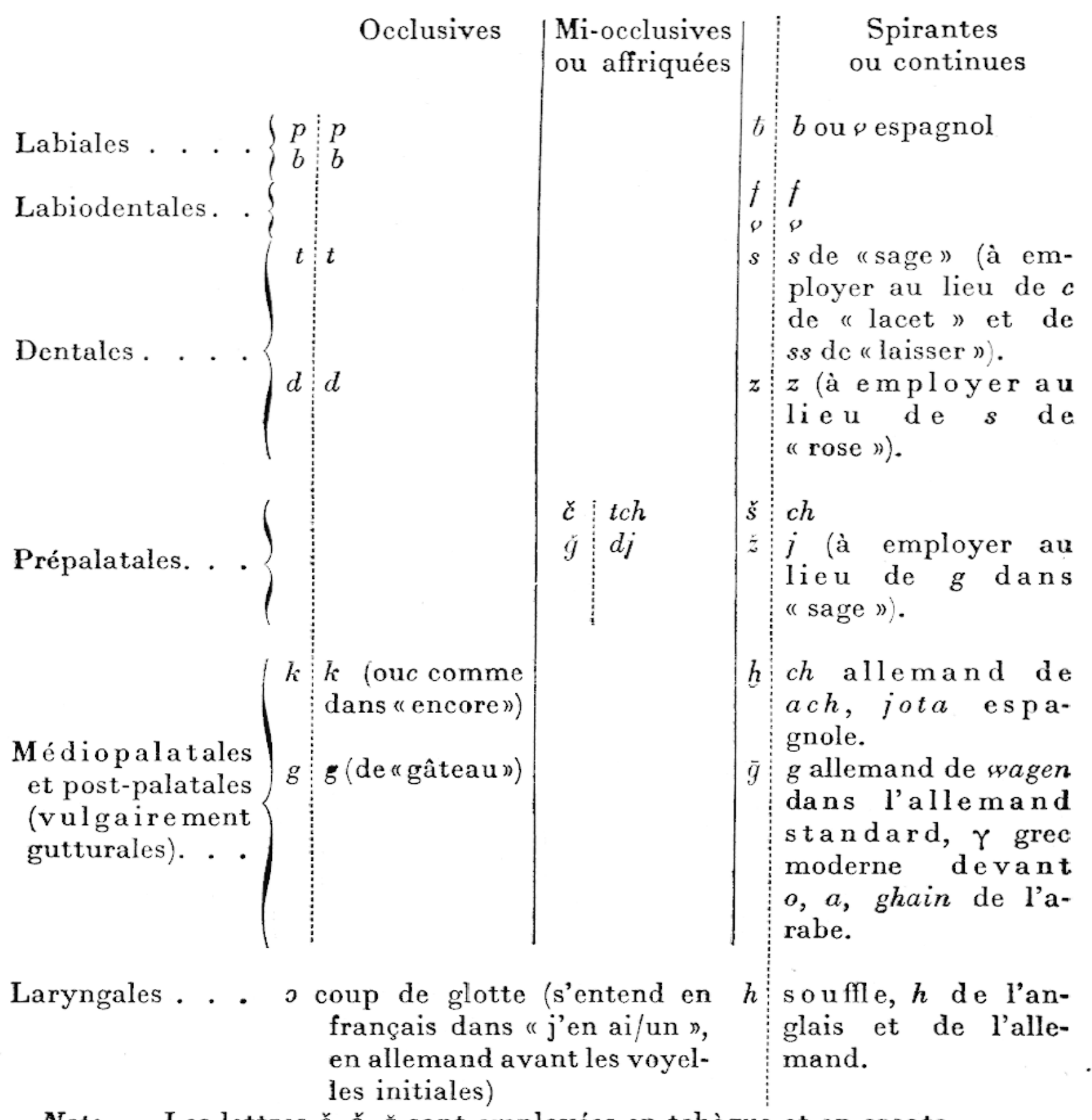

Note. - Les lettres $\check{c}, \breve{s}$, $\breve{z}$ sont employées en tchèque et en croate.

\section{Consonnes liquides.}

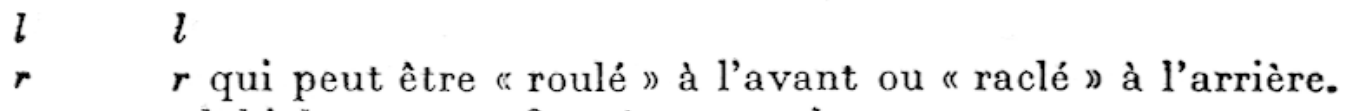

R $\quad r$ labial, souvent figuré comme brrr.

Consonnes nasales.

Labiale. . . . . . . . . . . m $m$

Dentale. . . . . . . . . $n$ n

Prépalatale........... $\hat{n} \quad g n$ 
La mouillure d'une consonne, c'est-à-dire la présence à sa suite d'un très léger $y$ est notée par un apex ou apostrophe rectiligne $: s^{\prime}$.

L'allongement d'une voyelle est marqué par une barre au-dessus : $\bar{a}$.

Un élément réduit est écrit au-dessus de la ligne : $p^{\mathrm{r}} a, a^{\mathrm{t}} a, p^{\mathrm{i}} t i$.

\section{LES CONDITIONS DE L'ÉTUdE}

\section{La société enfants-entourage.}

Le langage s'est développé chez les humains en société. Le nouveau-né est dès sa naissance dans la société de ses aînés pourvus du langage, et subit leur influence.

Ce n'est que dans des cas très rares que cette règle souffre des exceptions. L'infirme sourd, ou même le sourd-aveugle, a des contacts sociaux avec des parlants. Dans la société humaine, seul l'enfant séquestré par des parents criminels est privé des contacts normaux. On n'a pas fait d'expérience sous contrôle d'élevage d'enfants bien soignés mais soustraits à la communication, l'expérimentation pouvant comporter l'isolement complet ou celui de deux ou plusieurs enfants. Les quelques cas connus, trop mal observés, d'enfants enlevés et élevés par des animaux sauvages semblent comporter au moins partiellement l'acquisition du langage rudimentaire de ces animaux.

Or, dans toute société, il y a action et réaction des individus, d'autant plus marquée que ces individus sont de catégories différentes. Si les parents, et d'une manière générale l'entourage, agissent sur l'enfant, l'influence de celui-ci se fait sentir en retour.

De manière plus ou moins consciente, les adultes et les enfants aînés s'accommodent à l'enfant, de manières variées, soit pour le calmer, soit pour l'exciter à la gaieté, soit pour lui enseigner quelque chose : comportement, ou élément d'expression. Dans une plus ou moins grande mesure, "ils se mettent à sa portée " par des simplifications, et par des imitations de ce qu'il fait lui-même; ces imitations peuvent prendre le caractère d'amusement, quelquefois de contagion inconsciente amenant des sortes de régressions chez des enfants aînés avec peu de différence d'âge.

Il ne suffit donc pas d'enregistrer un maximum d'observations sur l'enfant, il faut aussi noter constamment (et enregistrer mécaniquement si possible) tout ce qui concerne l'entourage. Il faut avouer que jusqu'à présent cette obligation a été très mal observée; pour mon compte, je constate humblement dans mes notes le manque de beaucoup d'indications qui seraient utiles ou même indispensables. En réalité il est très difficile de tenir compte de tous les contacts; en dehors de ceux, qui sont habituels, des parents, frères et sœurs, grands-parents ou oncles et tantes, gardiennes et soigneuses habituelles, bien des relations 
occasionnelles peuvent avoir des conséquences : aussi faut-il ne pas oublier qu'elles existent.

Il faut surtout être conscients de certaines conditions générales, qui dirigeront les observations.

Dès le début de l'existence du petit d'homme, incapable de presque tout sans apprentissage, les adultes se trouvent comme avec des animaux domestiques, plus qu'avec ces animaux, en attitude de dressage.

Dressage utile, ou nécessaire, ou seulement distrayant, et qui s'exerce par beaucoup de moyens dont la parole n'est qu'un entre plusieurs.

D'une part, dès le début, on escompte plus ou moins consciemment l'aboutissement qu'est l'homme pensant-parlant; d'autre part on va au plus pressé et de proche en proche, par des actions appropriées à un animal supérieur qui n'est pas encore précisément l'homme; on fait toutefois constamment confiance à la faculté d'imitation, faculté propre à l'homme. (Rappelons que, contrairement à une fausse connaissance invétérée, les singes n'imitent pas normalement; il n'y a des cas exceptionnels de reproduction d'une conduite observée que chez des singes supérieurs).

Au cours des actions variées d'un dressage complexe, l'adulte adopte des attitudes spéciales. Par exemple, pour attirer l'attention de l'enfant, calmer ses pleurs, provoquer son sourire, etc., il exagère et multiplie des extériorisations normales comme le sourire lui-même ou des chantonnements. Il se sert aussi de moyens pas ou peu employés entre adultes, et plus ou moins en usage dans les communications avec les animaux : claquements de mains et de doigts, claquements de langue; de plus, ce qui concerne spécialement le bébé : bercement, quelquefois élévation en l'air, chatouillement et les procédés divers pour inciter à uriner et déféquer.

Quand il s'agit de provoquer l'imitation, toute une gamme de propositions entre un jeu, qui ira des gestes simples aux discours.

Il faut tenir compte aussi de toutes les attitudes de jeu prises soit pour les apprendre à l'enfant, soit pour y participer avec lui quand il les sait (faire des colonnes de cubes ou des pâtés), soit de manière à constituer un ensemble avec lui (porter sur le dos, sur les épaules).

Lorsqu'on en est à l'apprentissage du langage parlé, les conduites sont extrêmement variées. On peut admettre qu'on n'arrive jamais à parler à l'enfant qui ne sait pas encore vraiment parler tout à fait comme on parle à un enfant plus grand ou à un adulte.

Il ne suffit pas ici de savoir si les parents emploient ou non le langage " bébé ", avec abondance de mots enfantins à redoublement du type dodo. Il faut aussi savoir si les parents et le reste de l'entourage "bêtifient " plus ou moins, et si, pour se faire mieux comprendre et imiter, ils simplifient plus ou moins la grammaire normale de la langue. Par exemple : tel parent français propose-t-il à son enfant les substantifs nus ou avec article ou autre détermination? (Ainsi nez ou le nez, ou 
ton nez; chien ou le chien ou un chien?) Lorsque l'enfant commence a parler de lui à la $3^{e}$ personne (Bébé fait ceci), les parents emploient-ils la même forme, ou le tutoiement; et comment parlent-ils d'eux-mêmes et de tiers?

En somme pour bien juger du développement mental en général, il faudrait savoir jour par jour, heure après heure, ce qu'on fait avec lui et ce qu'on lui dit.

Suivant les coutumes de différents pays et de divers milieux, suivant les volontés conscientes ou les impulsions de l'entourage, on enseigne plus ou moins le langage à l'enfant, on rectifie ou non ce qu'il dit, on le serine plus ou moins : il en résulte des variations plus ou moins marquées dans les rythmes el les modes d'acquisition.

Étant données les facultés d'observation, de mémoire et d'imitation, il serait bon de savoir aussi tout ce qu'on fait et dit devant lui sans tenir compte de sa présence. Se rappeler que la littérature comporte des exemples frappants d'enfants commençant à parler avec l'accent d'une personne étrangère quittée depuis plusieurs mois ou même dans une langue dont leur entourage n'use pas, mais qui était celle d'un pays habité précédemment (1).

Le journal complet d'une existence de petit enfant doit noter, naturellement, les personnes, les objets, les paysages qu'il est amené à voir, les moyens de locomotion employés, etc.

Il faudrait enfin noter quel est l'ensemble de représentations offertes à l'enfant : jouets figurant en réduction des objets, des animaux, des personnes; images de toute espèce (y compris celles qui sont au mur, ou qu'on voit en se promenant). Savoir aussi quel est ou n'est pas l'emploi du miroir.

Tels sont les principaux traits dont doit se composer le tableau de l'ambiance sociale.

\section{La croissance de l'enfant et le langage.}

L'acquisition du langage s'insère dans la croissance et comporte en elle-même une succession, avec accroissement des possibilités et des efficacités.

Si l'ordre est fixe dans la croissance, sauf interruptions ou régressions accidentelles, les temps d'apparition et les durées des différents éléments, donc les synchronismes, varient dans de fortes proportions. Pour le langage comme pour le reste, il conviendra de rechercher les moyennes générales et par catégories (sexe, etc.) et d'autre part les circonstances et les conditions du plus grand nombre possible de cas individuels.

Les faits à observer sont multiples.

Il faut tenir compte de la santé générale de l'enfant : accroissement

11) Loopold. 111. p. 166. 
de poids et de taille, exercices réguliers de la digestion et de la respiration - on devrait dire aussi de la circulation et de l'influx nerveux.

Comme le langage comporte tout un agencement d'exercices musculaires délicats, tout ce qui est tonicité et mouvement est intéressant : agitations de la tête, du tronc, des bras et des jambes de l'enfant couché et porté, dont certaines peuvent être liées à des sensations et plus ou moins provoquées; plus tard, apprentissage (avec dressage plus ou moins forcé) de la station debout et de la marche; se souvenir que l'âge de la réussite varie couramment du simple au double sur la première période de 18 mois (c'est-à-dire précoce à 9 mois, tardive à 18) et qu'on a trouvé des enfants élevés par des bêtes n'ayant appris qu'une marche à quatre pattes; celle-ci est une étape habituelle mais non générale chez l'enfant dans la société normale, pour des raisons combinées de tonicité et de besoins et désirs, y compris ceux du jeu, de la curiosité et de la sociabilité.

D'une manière générale, tenir toujours compte de l'exercice, teinté ou non de jeu.

Comme le langage progresse avec l'intelligence, il faut essayer de dater les exercices des sens : perceptions et réactions qui s'ensuivent, surtout pour la vue et l'audition chez l'enfant normal. Une sensation tactile intervient sans doute dans les exercices du larynx.

La succession d'apparitions d'activités toutes sonores (sauf le sourire) de la gorge et de la bouche (accessoirement du nez), est exposée ci-après avec des datations très approximatives.

Pleurs, dès la naissance. Mais il ne suffit pas de les mentionner ainsi; il faudrait en étudier toutes les variétés, avec et sans sanglot, et dater l'apparition des larmes.

Sourire. Autour de 1-2 mois. Sans doute, intermittence de son usage, quand le rire apparaît.

Émissions vocaliques isolées. Même période.

Gazouillis (ou gazouillement, babil, lallation), d'abord voyelles ou principalement voyelles (1); puis principalement consonnes, à partir de 2 mois $1 / 2$; régressant vers 9 mois. (Par la suite, subsiste à certains moments restreints de la journée, en marge de l'acquisition du langage.)

Rire aux éclats au cours du $3^{\mathrm{e}}$ mois.

Cris, gémissements et grognements variés, éventuellement clics, servant à des communications. Début vers 10 mois.

Emploi volontaire d'émissions significatives généralement composées à la fois de voyelle et consonne. Début vers 1.0 mois.

Les bâillements et éternuements apparaissent dès le début, ainsi que dęs halètements et des souffles du nez ou certains raclements en cas de rhume et le hoquet; la toux proprement dite est postérieure. A quel moment apparaît le soupir?

(1) Poriode qualifibo par Leopold de - noveoulemeot o fo cooing i) Voir II, p. 139. 
La croissance mentale, non séparable de la croissance et de la différenciation des centres nerveux supérieurs, commence dès la formation de ces centres. Elle n'est que partiellement observable. L'observation est facilitée, tout en restant très limitée, lorsque le stade du langage complet est atteint.

Le caractère d'abord rudimentaire de ce langage et sa progression relativement lente sont l'indice de la lenteur de la croissance des diverses sortes d'activité mentale, notamment de la réflexion et de l'abstraction.

\section{Les moyens de communication de l'adulte.}

Pour bien observer la période d'acquisition du langage, il faut connaître et considérer méthodiquement l'état d'aboutissement.

Si on définit le langage comme instrument de communication, on doit estimer que tout élément qui sert à la communication est partie de langage, même s'il peut apparaître éventuellement hors langage, et sans tenir compte s'il est sonore ou non. En conséquence, disons-le expressément tout de suite, un geste employé au lieu d'une émission de voix est tout autant que cette émission un élément de langage.

Inversement un élément vocal ou un geste qui sert habituellement de communication peut être employé de manière réflexe sans volonté de communication, ou à titre d'exercice et de jeu, sans intention sociable.

Les éléments de communication dont on dispose sont variés; dans les descriptions de langue, on en dissimule généralement une partie, en ne citant que les mots proprement dits.

Ce qui suit concerne les conditions de l'adulte normal.

a) Des réflexes sonores peuvent remplacer une phrase (étant entendu qu'une phrase peut consister en un seul mot), ainsi principalement le rire, aussi les pleurs, les sanglots, les soupirs. Ces éléments peuvent être lexicalisés, étant employés volontairement à la place d'une phrase, soit par émission forcée imitant plus ou moins bien l'émission naturelle, soit par des transpositions en groupes de phonèmes habituels ou exceptionnels de la langue qu'on classe comme interjections. Pour l'usage français, mentionnons spécialement la toux ou le toussotement, employés comme avertissement ou mise en garde, et les figurations écrites hem, hum.

b) Parmi les réflexes, il y a des émissions de voix qui sont des cris (douleur, surprise, joie) et des souffles sans voix (surtout dégoût, admiration). Ils sont employés dans les mêmes conditions que les éléments précédents, avec plus de variété comme exclamations. Ils sont lexicalisés occasionnellement : souffle des lèvres admiratif ou méprisant, imitation de crachement de l'arrière-bouche dépréciatif, etc. Dans la langue écrite $p f, p f f, p f i t, p f u t, a h, o h$, ho, etc. 
c) Une catégorie des gémissements et grognements ou murmures comporte surtout, dans les langues européennes, des émissions nasales : à bouche fermée $m$, à bouche ouverte $\tilde{e}$ (in de l'orthographe française); susceptibles d'intonations qui sont les mêmes que celles des phrases articulées, elles servent à exprimer toutes sortes de nuances d'approbation et d'aquiescement, de négation et de refus, de doute, d'interrogation. Dans le français écrit, hein et quelquefois meuh-meuh de doute (1). L'occlusive glottale intervient surtout dans les langues qui ont des phonèmes laryngaux, ainsi arabe maghribin $0 \tilde{e} \rho \tilde{e}$ " non ".

d) Les clics ou bruits de succion, tels qu'il s'en produit dans la mastication et qui servent normalement de consonne dans des langues de l'Afrique méridionale, sont à ranger à côté des grognements.

Le clic labial ou bruit de baiser est un des plus usuels. Le clic dental de contrariété est quelquefois figuré par écrit comme $t$, ou tutt. Émis une seule fois (ou deux), c'est une négation usuelle sur les bords de la Méditerranée, en particulier en Algérie, dans les langues européennes aussi bien qu'en arabe.

e) Les mots, généralement groupés en phrases, sont composés de phonèmes, c'est-à-dire de consonnes et de voyelles en nombre limité, dont chaque langue a un stock particulier; ils sont généralement marqués d'un accent (quelquefois un scul accent pour un groupe). Ils comportent soit dans leur constitution soit par leurs agencements réciproques l'ensemble des caractéristiques grammaticales.

f) La mimique comprend des petits déplacements musculaires de la face (mines), des déplacements de la tête, des bras et des mains, plus rarement du tronc ou de ses parties et des jambes (gestes). Tous ces éléments peuvent remplacer des phrases; ils ne sont pas figurés dans le vocabulaire, mais ils sont décrits dans les romans ou indiqués comme jeux de scène dans des pièces de théâtre : sourire, clignement d'œil, moue; oui et non de la tête; pointage du doigt, dressage de poing; haussement d'épaules; tapage du pied, etc. Très souvent ces mines ou gestes accompagnent une phrase, au lieu de la remplacer.

g) Pour mémoire : l'écriture; les figurations dessinées; les moyens sonores de reproduction de la voix.

L'homme silencieux et immobile ne cesse pas pour autant - mais à partir de quel âge? - d'employer le langage en mots : c'est ce qu'on appelle le langage intérieur, qui peut ne s'accompagner d'aucune amorce d'activité musculaire ni de sentiment d'audition ou de vision (état normal au moins chez un intellectuel de civilisation supérieure), et qui est l'instrument de la réflexion individuelle ainsi que de la lecture muette.

D'autre part l'homme occupe une partie de son activité vocale, géné-

(1) J. Manozzeau. Aspects du frangais, 1950, p. 23-24. Marie-Louise Mïrler-Hauser. La Mise en relief d'une idé en français moderne, 1943, p. 144-147. 
ralement en accompagnement de diverses activités qui ne comportent pas de communication, par des chants ou chantonnements avec ou sans paroles, ou par le sifflement. Mentionnons : l'individu à sa toilette, la femme qui coud, le cultivateur qui laboure. I.a musique n'est pas indispensable à ces manifestations : la récitation peut jouer le même rôle. Noter qu'en général l'usage du langage intérieur se poursuit en même temps.

A ce propos, on peut mentionner que des mots peuvent apparaitre en tant qu'émissions non significatives au sortir du sommeil, remplacant des gémissements et grognements ou l'étirement, ou s'y ajoutant.

Enfin il y a encore à mentionner des suites longues d'émissions monotones sans signification précise. Elles sont émises occasionnellement ou habituellement par certains individus, soit comme monologue solitaire, soit à l'adresse de jeunes enfants ou d'animaux, en tant qu'agacerie. On peut donner comme exemple, exceptionnel en littérature, dans Colette, Bella Vista, éd. Ferenczi, 1937, p. 69, la figuration en huisipisi, huisipisi d'une suite sifflante, émise par un individu qui agace une chienne.

De tous les moyens de communication énumérés (avec certains de leurs usages dérivés), nous connaissons en gros les proportions d'usage chez l'homme moderne normal des civilisations à la fois industrialisées et le plus diversement intellectualisées. Mais qu'en est-il dans des civilisations plus frustes et quel a été le point de départ chez les plus anciens hommes?

Chez les hommes actucls, il y a bien des petites différences et des usages locaux : partout le langage parlé est l'instrument essentiel de communication, nulle part il n'est exclusif.

Pour l'origine, il n'y a aucune raison de supposer un premier langage exclusivement ou presque exclusivement gestuel, comme l'ont fait certains, ni non plus, comme l'ont fait d'autres, un premier langage dessiné. Pour la science moderne évolutionniste, à défaut d'autres arguments, la prédominance des manifestations vocales chez les animaux les plus proches de l'homme suffit pour écarter l'idée d'un caractère secondaire de la paróle.

\section{Description sommaire de l'acquisition}

Note liminaire.

Il est tenté ici un essai de mise en place d'une description, avec mise a profit des observations personnelles ou personnellement communiquées à l'auteur, et compte tenu des observations publiées par divers auteurs et de leurs interprétations. La discussion est réduite au minimum. 
La question de la part d'expression des besoins et des débuts de sociabilité dans les pleurs et mouvements de la première période n'est pas traitée.

Autant que possible, les phénomènes ont été discernés et distingués dans l'exposé; mais aussi il a été fait effort pour montrer les enchevêtrements, les chevauchements de transformations partielles, les connexions et les interactions. Le lecteur devra compléter, en se reportant d'une division à l'autre pour rétablir les synchronismes.

Les moyens sont malheureusement très insuffisants. Le matériel utilisé est réduit : l'observation des enfants a été et est en partie intermittente : pour Fr., il faut tenir compte que je n'ai disposé, en temps de guerre, que des permissions; les notes de ma femme, utiles, ne sont pas très abondantes; les petits-fils $J$. et $J$. L. ne sont qu'épisodiquement avec moi, et les parents ne prennent pas de notes serrées. D'ailleurs, même aux périodes de présence, je n'ai pas pu et ne peux pas donner tout mon temps à l'observation. Je n'ai pas de magnétophone.

Tels quels mon matériel et quelques notes reçues d'autres parents mériteraient d'être publiés complètement; c'est exclu actuellement surtout faute de temps pour la mise au net, parmi beaucoup d'autres occupations. (1)

Il serait désirable qu'on possède les documents les plus complets possible donnant des tableaux jour par jour, avec distinction des moments de la journée, ceci sinon tous les jours, au moins à des jours rapprochés (l'observateur averti notant dans l'intervalle les faits saillants). Il faudrait pouvoir aussi indiquer les densités d'emploi.

Le moyen d'avenir sera le magnétophone fonctionnant à longueur de journée, ou le cinéma sonore, et autres inventions à prévoir.

Pour une étude complète, il cst nécessaire de prendre conscience des synchronismes, et de noter tant les disparitions ou atténuations que les apparitions et augmentations.

A cet effet la transcription des fiches en alignement, ou l'audition de la bande de magnétophone, ne fournissent qu'un matériel brut : on ne peut comprendre quelque chose qu'en procédant à son élaboration, toutefois sans excès de dissociations.

Il faut souhaiter que dès que ce sera possible on emploie judicieusement pour l'élaboration et pour l'exposition des résultats les inventions modernes, déjà existantes et à venir. Au lieu d'un pauvre exposé écrit réalisé tant bien que mal comme celui qui va suivre, on devrait pouvoir offrir dès maintenant des graphiques colorés animés et des sélections sonores.

Pour une publication complète, avec les moyens ordinaires de l'écriture, on pourrait conseiller :

(1) Considérer les dates comparées des observations et des publications de Leopold et Grégoiro. 
a). Un tableau d'ensemble numéroté, avec les définitions et un minimum d'exemples. (Un tel tableau deviendrait inutile dans les ouvrages particuliers, si un plan standard était adopté).

b) La transcription intégrale du journal tenu, avec des divisions suivant le tableau, chaque fait étant suivi d'un chiffre renvoyant à une case de ce tableau (lorsqu'il ne concorderait avec les divisions adoptées). Des signes spéciaux pourraient indiquer les premières apparitions.

Ce serait plus clair si on pouvait employer des couleurs.

Dans le présent mémoire, les exemples ont été donnés nombreux, mais sans épuiser la documentation; la physionomie des sujets dans leur comportement général n'apparaît que peu.

Les observations ont porté sur des enfants d'intellectuels, élevés par leurs parents presque exclusivement. On se demandera si de tels enfants sont ou non plus précoces que des enfants grandissant dans d'autres conditions. Ce n'est pas probable : dès maintenant on peut constater des retards particls chez une partie des sujets. Mais la multiplication des enquêtes pourra seule fournir un commencement de réponse solide. $\mathrm{Au}$ total l'essentiel du langage est toujours acquis dans la petite enfance, quelles que soient les inégalités individuelles, dues ou non aux circonstances sociales ${ }^{1}$.

\section{A. - Le gazouillis.}

Le gazouillis est un phénomène curieux sur lequel il ne faut pas se tromper. Il paraît être essentiellement $d u$ caractère exercice, principalement exercice du larynx, accessoirement de la langue et des lèvres. Y a-t-il une relation avec la respiration? Dans quelle mesure doit-on lui attribuer dès le début un caractère de jeu, s'exerçant en euphorie, et avec des sensations agréables? Il n'a pas de caractère de socialibité.

Le gazouillis paraît chez les sourds de naissance, pour qui il ne peut pas comporter de contrôle auditif.

D'ailleurs, il n'imite pas des sons émis par les adultes. Il comporte normalement une assez grande variété et, au moins dans les langues où il a été observé, certains sons qui ne font pas partie des phonétismes de ces langues, mais de loin pas toutes les articulations possibles.

Après quelques mois, le gazouillis se restreint, avec des péripéties variées.

L'apparition du langage imité des adultes bénéficie évidemment des exercices musculaires réalisés dans le gazouillis, mais ne se fait aucune-

(1) Témoignage d'un père, intellectuel lui aussi, sur ses trois enfants : le premier a parlé à un

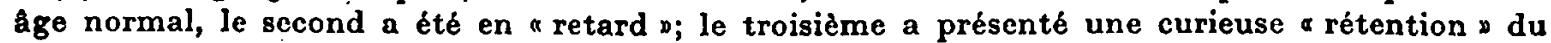
angage, ne parlant pratiquement pas jusqu'd cinq ans, et a alors rapidement raltrapó le retard. 
ment par une " sélection" dans les sons de celui-ci. Peut-être pourrait-on noter inversement que le gazouillis de cette période a en partie au moins le caractère d'exercices supplémentaires sur les premières articulations imitées. Une observation de la terminaison du gazouillis chez les sourdsmuets serait très utile pour comparaison. Il faudrait savoir si ses manifestations postérieures sont suppléées par quelque chose.

Il est probable que les premières émissions d'une voyelle de timbre sensiblement a ( $e$ muet du français) ou proche de $\ddot{a}$ ( $a$ tendant vers $e$ [è du français]) sont des amorces du gazouillis, et non des premières communications, mais il y a peut-être les deux (1).

Fr. 2 1/2. Dit quelquefois a prolongé (à la même date noté qu'il fait un éclat en riant).

L. $11 / 4$. Des $\ddot{a}$, à l'état de repos.

Chr. 1 1/2. Une note dit qu'elle émet plusieurs a [en se voyant dans la glace; fait peu vraisemblable, les enfants ne regardant pas vraiment en général à cet âge].

2. Une voyelle a (suivie d'un léger raclement sourd).

3. Suites de a, donnant l'impression d'une communication.

J. 2. Des sortes de $\ddot{a}$, une fois au moins en écho à une proposition analogue; un souffle $h$; à la même date des sourires.

$21 / 4$. Des $\ell$ dont certains semblent inspirés; des souffles $h$ (et sans doute aussi des inspirations).

J. L. 3. Des a; en même temps des cris d'aise, très aigus.

Le gazouillis s'observe normalement au lit, le petit étant à l'aise, notamment au cours d'une bonne digestion, avant de s'endormir et ne voyant pas les grandes personnes.

Fr. $51 / 2$. Note imprécise : gazouillis abondant, articulations variées.

9. $t \ddot{a}$ dä ätä, ya, pf(a), bv (sans voyelle) ma ma ma ma mam (a proche de a).

Autre note, d'un des jours suivants : dominance de $t \ddot{a}, d \ddot{a}$, rarement $g^{\mathrm{y}} \ddot{a}$; pas d'occlusives plus reculées, mais spirante sonore vélaire, sorte de $\bar{g}$ ( $g$ spirant) ou $r$ d'arrière, suivi de $\dot{a}$ ( $a$ profond); clic latéral, noté $k l$, suivi de $a ̈$; exceptionnellement une voyelle autre que $\ddot{a}, \partial$, dans $b u$ ( $u=o u$ français); labiales rares, cependant des mämämä, päpäpä. (A la même époque a commencé l'imitation de syllabes proposées).

$91 / 4$. Le gazouillis spontané du matin au lit se fait sur un ton plus aigu que les essais d'articulation par imitation.

Suites : bababa, nanana.

11. Depuis quelques jours, tout le gazouillis se fait sur des exercices de labiales: waswawa, tatata (t) est le $b$ spirant espagnol, $\diamond$ bilabial), mamama, mamama, papa (rare), na (rare).

(Dans le même temps, il apprend à comprendre papa; l'émission mãmã a quelquefois l'air d'être un appel, mais non précisé pour la mère.)

18. (Notations de la mère.) ptié, mné, pton, mané, chté, cron.

(1) Póriode de * roucoulement *. Voir ci-dessus p. 191, note. Pour la communication, ci-dessous p. 215 . 
L. 2 1/4. Apparition des premiers $r$ d'arrière.

2 1/2. Gazouillis fréquent aux heures calmes; bruits de gorge, claquements de langue. Voyelles a avec légère frication laryngale.

Puis : long gazouillis matinal; dominance de $g$ suivi d'une vibration linguale ressemblant à $l$ ou $r$.

3. Dominance de $g$ et $r$ d'arrière. Quelques $t$ et $d$, un essai de labiale. [En même temps, premiers rires aux éclats, provoqués par secouement, ou par une gesticulation en sa présence.]

4. Abondance de gazouillis en " sons modulés", peu de consonnes.

5. Une note dit, comme résumé d'une période; surtout cris et chantis, voyelles peu déterminables; consonnes rares, mais assez variées, palatales, dentales, labiales.

6. Apparition des nasalcs, d'abord sporadiques : nänä, mämä.

6 1/2. amba-amämä-anänä, mämä (assez fréquent) mämämä (rare).

(Un autre jour :) sortes de añan $\tilde{a}(\bar{n}=g n$ du français), quelques $t a$.

$63 / 4$. Le gazouillis satisfait est surtout sur $t \ddot{a}$ en sortant un peu la langue; les $m a$ semblent indiquer une légère contrariété.

7. Une note dit que les exercices sont laryngaux (presque plus d'articulations buccales); mais quelques jours après : recommence à faire des exercices de labiales et dentales. Puis on note une plus grande différenciation des voyelles $(b \ell b \ell, v o, p a)$.

7 1/4. Abondance de dentales.

8. Note résumée pour une période : peu d'articulations de consonnes buccales, voyelles, clics, claquements de lèvres (bruits de baiser, de pet, etc.), bruits laryngaux.

$81 / 2$. Reprise des consonnes $(t, n, m)$, continuation des bruits de lèvres.

A partir de cette date, je fais un choix; les observations sur les exercices (dont les moments ne sont pas précisés) s'entremêlent avec celles sur les dressages et sur les débuts de langage parlé.

9. Des papa, mama, amama, une fois un $r$ labial réussi.

$91 / 4$. Clics médians (claquements de langue); des $p \leftrightarrow q a, b s p a$.

$121 / 2$. Fréquents : mamama, dädädä.

13. Copie textuelle d'une note : "Semble dire beaucoup de choses que nous ne comprenons pas; dans la variété de ses exercices, il semble y avoir des mots. Mais il y a aussi exercice et jeu - mécanique à moitié voulue, moitié mal commandée; ainsi (a)papapapapa sur la base papa (qu'elle commence a répéter sur demande, mais ne possède pas comme mot spontané). "

Quelques jours après, on note des espèces de träträträ.

15 1/4. Dernière notation d'exercice, d'ailleurs isolée : lololo.

Chr. 2 1/2. Gazouillements à sons variés : $\ddot{o}$ (eu du français) suivi de $\varepsilon$ et inversement $\varepsilon \ddot{o}:$ vagues $y, u p, l$.

$51 / 2$. Gazouillements variés : séries de $h$ (ach-laut de l'allemand, spirante post-palatale sourde) forts et prolongés, seulement quelquefois accompagnés de voyelles; aussi la sonore $\bar{g}$; exercices labiaux surtout en ba; des cris de gorge, certains genre sanglot (occlusions glottales?).

Peu de jours après : beaucoup de $b \hookleftarrow a$, quelques $l a ̈$, espèces de bla. Plus tard encore, on note un $t a$.

6. Noté un aga. 
$61 / 4$. Suites papapa, séries de $b$ profonds et violents.

7. Suites : gagaga (et kga-kga).

8. Suites : papa papa.

$81 / 2$. Suites : adad, adada (en partie avec des $d$ assourdis); un ton triste s'exprime par dędęde.

9. Suites : $n \ddot{a} n \ddot{a}$.

11. Périodes du type at et du type bab; des pf; aussi des ga, gס̃.

(Entre temps apparitions d'articulations au moins rudimentaires de mots répétés ou spontanés.)

$113 / 4$. Suites de alala.

13. Une note dit : gazouillage augmenté et précisé d'articulation; apparences de conversation; articulation usuelle : bŭye bŭye.

14. Exercices de roulements de langue : $g^{\mathrm{r}} \boldsymbol{i}$, gidira, ridira.

17. Dans le même genre galiga gii ( $\dot{n}=n g$ anglais), kitik ${ }^{\mathrm{r}} a$.

J. 2 1/4. Mouvements de langue, sans émission de voix; inspirations et expirations sur $\ddot{a} ;$ des $k$ profonds et suivis d'un léger souffle, sans voyelle; quelques amorces de clics.

(Par ailleurs, sourires.)

$23 / 4$. Dominances vocaliques.

3. Émissions fortes; espèces de ra (avec $r$ d'arrière), $\varphi$ bilabial prolongé.

$51 / 2$. Des eris et gloussements modulés; éclipse des exercices de consonnes. (Par ailleurs rires aux éclats.)

7. Des suites de ata où $\iota$ semble être obtenu avec l'arrière de la langue; des cris aigus.

9. Séries $\rightsquigarrow a a \ddot{\psi} a(\ddot{\psi}=$ français $u$ de puis), tatja.

10. Séries : ya, gya, ata, ada.

14. Séries : tatata et $g^{y} a g^{y} a g^{y} a$ ou approchants, avec la voix plus ou moins forcée.

Quelques jours après des aya, dadda et des awa, des ka, encore après : s'amuse à n (labial) et aussi à un clic médian qui lui a été proposé.

(Par ailleurs débuts du langage parlé.)

A partir de 17 mois, apprend des chansons, désormais les répète de plus en plus au licu de gazouillis, ou entre des gazouillis.

Il dit souvent, avec plus ou moins de volubilité, des " choses qui ne veulent rien dire "; exercices d'élocution, semble-t-il, sur des sons ordinaires du français; aussi, manières d' "occuper le temps" par un jeu vocal. Noté par sa grand-mère à $243 / 4$ lakapa atama azana olükñ dikatan (Les psychologues devront peut-être comparer les gribouillis de l'âge plus avancé.) bulles.

J. L. 5. Voyelles à grande voix; des $\bar{g}$ (g spirant) et gr; quelques $p f$, et

7. Des ata.

8. Des ata et aga.

9. Des agaga, ya; des sortes de $h$ et $\bar{g}$; $q g i$; émissions de voix forcée.

10. Toutes sortes de sons difficiles à noter, surtout des post-palatales; quelques labiales sourdes : $p f$. 
21 1/2. Noté dans un gazouillis : à peu près lalalada, alors que $l$ est encore mal établi dans son langage parlé.

24. Dans le gazouillis au lit, soit au réveil du matin, soit avant de s'endormir à la sieste, il semble qu'il fait une sorte de revue du vocabulaire en mots connus, comme maman, papa, entremêlé et surtuut suivi d'émissions sans signification, où il n'y aurait pas d'articulations non normales (?). En dehors du lit, use peu, peut-être pas du tout, des émissions dénuées de sens.

Dans l'ensemble, les observations fragmentaires sur les cinq enfants sont concordantes; par ailleurs je n'ai pas constaté de faits contraires dans les ouvrages consultés.

Il y aurait donc une première période d'exercices vocaux actifs, à un moment où par ailleurs le langage parlé n'est pas encore employé. Les sons étrangers au français qui sont plus ou moins fréquents sont les spirantes vélaires d'une part, un complément de la série labiale d'autre part; plus rares, certains éléments laryngaux.

Dans une seconde période, où déjà est apparu le langage de communication sous ses différentes formes, le gazouillis servirait en partie d'exercice sur les sons normaux de la langue, en présentant à l'occasion des sons non encore acquis dans le langage parlé.

D'autre part, il aurait pour fonction d'occuper "le champ d'élocution " (et plus ou moins le " champ de conscience "?) à certains moments. Ceci concurremment avec le chant chez les individus qui savent en user.

On devra sans doute se demander dans quelle mesure la gesticulation vocale du jeune enfant au cours de l'acquisition du langage n'est pas en grande partie au moins de la pensée à haute voix, à un moment où le langage intérieur n'est pas encore installé. (Se rappeler que chez l'adulte les réminiscences et réflexions habituellement muettes s'extériorisent dans des moments de trouble (1).)

\section{B. - Le dressage extralinguistique; les jeux.}

La vie commune des jeunes et des aînés (les enfants qui ne sont plus des nourrissons) comporte des séries de dressages, où sont compris les jeux et les premiers rapports de politesse. (Des premières propositions de mots à reproduire par imitation il sera question plus loin.)

Le dressage s'adresse successivement à des possibilités de l'être vivant de plus en plus développées et de caractère progressivement plus " élevé ", c'est-à-dire de plus en plus proches de la pensée raisonnante. Le dressage n'est possible dans ses différentes parties qu'à mesure que la croissance permet certaines manifestations, mais aussi il permet lui-même des accélérations et des stabilisations dans la mesure des possibilités.

(1) Voir Norman, cité p. 184. 
Les premières habitudes inculquées (repas, sommeil, excrétions) concernent la vie végétative.

Mais vite on rencontre et on dépasse le niveau des animaux supérieurs, avec usage de facultés d'attention, de reconnaissance, de mémoire consciente, enfin de raisonnement, auxquelles il faut joindre les besoins et facultés de jeu et de sociabilité : tous ces termes sont lancés ici avec plus ou moins de précision : c'est aux psychologues qu'il appartient de travailler la matière, notamment en développant la psychologie comparée. Au total, il s'agit d'observer comment se développent les premières techniques du corps et les débuts des techniques intellectuelles en dehors du langage.

Mais le dressage se faisant en grande partie au moyen précisément du langage, on rencontre presque dès le début la question de la compréhension de celui-ci, précédant son emploi par le sujet. Ce qui pour l'animal reste toujours un signal auquel il ne fait qu'obéir deviendra pour le petit d'homme un instrument qu'il possédera en commun avec le dresseur.

Le calmement des pleurs - dans la mesure où on ne laisse pas pleurer l'enfant précisément au cours de l'acquisition d'habitudes horaires et l'incitation au sommeil peuvent comporter des bercements, premiers exercices rythmiques (dont le roulement en voiture, dans un même sens ou alternatif, est une variante), et le chant, qui commence l'inscription dans le petit cerveau d'autres rythmes, accompagnés de mélodie et de l'articulation de la voix.

La provocation au sourire et au rire, lorsqu'ils apparaissent, comporte, de la part de l'adulte ou de l'enfant aîné, un usage plus ou moins développé des jeux de physionomie, de la gesticulation, de bruits variés, enfin de la parole. Autant de signaux qui peuvent s'inscrire plus ou moins clairement chez le sujet. Les défenses et intimidations comportent aussi des éléments de mimique, dont le principal est le signe de tête "non v (avec ou sans le mot).

Viennent ensuite des jeux, où la participation de l'enfant s'inscrit avec plus ou moins de retardement et augmente plus ou moins vite. Dans la mesure où cette participation est sollicitée, on ne lui demande pour commencer que des macro-mouvements, exigeant bien une première accommodation musculaire, mais par l'exercice de grands muscles, et sans finesse ni précision (ainsi les "au revoir " du bras); des habiletés par exercices de plus petits muscles, y compris ceux de la parole, peuvent se révéler plus ou moins précocement chez les sujets, suivant qu'ils sont plus ou moins doués; un dressage systématique peut naturellement obtenir des résultats spéciaux, comme avec des animaux.

Pour la comparaison avec les animaux, noter qu'on ne recourt pas normalement avec les enfants au simple appétit normal ou à la gourmandise comme moyen de dressage; cependant certains parents le font. Mais comme avec les animaux, quoique sans doute moins qu'avec 
- les animaux chasseurs, chien et chat, on rencontre l'aptitude au jeu, et plus qu'avec cux le désir de compagnie affectueuse.

L'introduction de la feinte et de la fiction dans le jeu est une affaire importante, qui voudrait de longs développements. Il n'y est fait ici que quelques allusions.

Les premiers gestes de sociabilité commandée dépassent en général le niveau de dressage habituel des animaux domestiques, bien qu'on puisse apprendre aussi à ceux-ci à demander poliment et à remercier. Les sentiments d'affection qui y sont plus ou moins liés ont leurs correspondants dans des manifestations naturelles de ces animaux.

Comme nos enfants n'ont jamais été tapés, il ne sera pas question ici de la crainte des coups.

Dans les tableaux synchroniques des manifestations de la vie enfantine, il faut tenir compte des acquisitions essentielles oũ le dressage n'est qu'un adjuvant à des exercices personnels de techniques du corps. Enumérons :

a) Le manger sous ses différentes formes, jusqu'au moment où l'enfant mange seul et plus ou moins proprement (on ne trouvera ci-dessous aucune note à ce sujet).

b) Le gouvernement des sphincters n'est pas noté non plus ci-dessous, malgré divers exemples concernant le pipi; les enfants observés n'ont pas présenté d'anomalies, et ont été dans -la moyenne, c'est-à-dire " demander à faire pipi ", de jour, autour de la fin de la deuxième année, assez longtemps après que le langage parlé est installé, et employé aे divers autres usages.

c) La station debout, puis la marche. Sa réalisation est indiquée dans la suite, inséréc dans les tableaux d'acquisition du langage.

En fait ces tableaux montrent que les dressages de marche, de premiers jeux et de premières marques de sociabilité sont postérieurs au stade propre du gazouillis et contemporains des premières propositions de mots à l'imitation de l'enfant et des premiers exercices de celui-ci pour en acquérir le maniement.

Rien de ce qui concerne le langage proprement dit n'est traité dans la présente division. Les jeux n'ont pas été notés complètement, il n'a été relevé que ce qui paraissait indiquer des faits de compréhension et des réactions caractéristiques.

Fr. Pas d'observation précoce. A 9 mois, il est noté qu'il comprend la défense parlée non et le geste de tête correspondant (rotation latérale avec léger balancement), y obéit quelquefois, et fait quelquefois lui-même le geste.

Les indications suivantes sont postérieures au début des manifestations linguistiques.

A 11 1/4, réagit à " écoute n, semble comprendre a donne 》. Joue spontanément à donner des objets en les mettant dans la main ou la bouche 
de sa mère ou de son père et à mettre sa main dans la leur pour qu'ils la serrent.

A 15. Fr. fait quelques pas.

La somme à $151 / 2$ est la suivante, sans qu'il y ait de notes sur les dates d'acquisition en détail. Sur commande : embrasse, caresse de la main, fait bravo, au revoir (du bras); fait coucou (il n'est pas dit comment) et " ah! le voilà " parlé sous la forme aaa ou swala. Se déplace sur son derrière.

A 16. Fr. marche seul. Fait mimi, coucou, au revoir. La fiction en actes est représentée : il berce dans ses bras un ours en peluche ou d'autres objets.

L. 7. Première notation, que je ne sais pas bien comment classer : me tend les bras quand je tends les mains pour la prendre.

$71 / 4$. Se tourne vers des personnes sur question : "Où est?... "

$71 / 2$. Joue souvent à se rejeter en arrière. Le fait quelquefois sur le signal " pouf ».

8. On lui offre un rond et une cuiller. Elle joue alternativement avec, posant et écartant l'un avant de prendre l'autre.

9. Ne réagit plus à : "où est papa?»; mais se tourne dans la direction voulue à la question " où est le pot?".

10. A l'invitation "fais coucou» cache sa tête avec sa robe; elle repousse à deux mains la tête de son frère Fr., qui se redresse, et elle rit aux éclats.

$103 / 4$. Essaie de se mettre un couvercle sur la tête (comme on le lui fait quelquefois).

11. Obéit à " couche-toi " (étant assise).

$111 / 2$. Joue à "coucou ", un a très aigu vaut " ah la voilà ". Fait bravo sur demande sans avoir été précisément dressée; quelquefois dit $b$ ou bu en même temps.

$113 / 4$. Sur l'invitation "pouf, pouf, pouf ", tape dans la main, en soufflant fort, ce qui est peut-être une imitation de "pouf ".

$121 / 4$. Ne fait le geste de bravo que spontanément, et sans émission d'accompagnement. (On ne dit pas pourquoi elle fait ce geste.)

$121 / 2$. Fait merci du bras (sur invitation ou non?).

13. Fait " au revoir » avec sa main sur invitation.

Met des objets sur sa tête pour faire chapeau.

Fait des colonnes de 5 et 6 cubes; la première réussie a été saluée par des cris de triomphe.

$131 / 2$. Une note de bilan dit que la compréhension est en progrès, ce qui est marqué en particulier avec réactions correctes à "montre ton œil, ta bouche, tes dents, tes cheveux, ton pied, ta main $n$.

A appris instantanément à envoyer un baiser, faire "vive... " (les bras en l'air), faire au revoir, etc. [Remarquer que "au revoir " a été noté auparavant et que le etc. est énigmatique.] Remarque intéressante : se rappelle bravo, semble avoir oublié merci.

Commence à faire des pas, étant tenue.

16. Sur demande, montre sur elle " cheveux, menton, yeux, nez, bouche, dents, langue, pied, main, front $"$, a oreille " pas toujours; confond ton oil

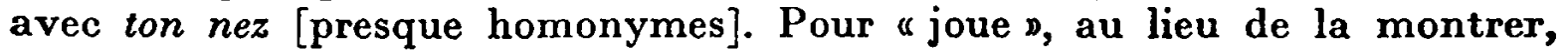
ouvre et ferme vivement ses mains. Plusieurs jours après on note que, tout en continuant ce mouvement de mains, elle montre sa joue. 
$161 / 4$. Sur demande de sa grand-mère "veux-tu faire joujou ", agite les mains comme elle avait fait pour " joue ": il y avait donc eu confusion de mots.

Par une autre confusion, met sa poupée dans son "dos" pour lui faire faire "dodo "; d'autre part elle la berce en disant to to to to.

Sa grand-mère dit "fais la diable "; elle agite les pieds et le corps.

16 1/2. A l'invitation : "prends l'air fâché ", fait une moue en fronçant les sourcils, et en se retenant de rire.

17 1/4. Chatouille, en émettant à peu près kritikri.

18. Sait embrasser.

19. La marche vient d'être acquise.

Chr. 9 1/2. Répond $\bar{a}$ à "coucou".

Refuse quelquefois un objet proposé, et en prend un autre.

14. Participe à " coucou " par une sorte de $a$; donc non progrès.

Tend la main en geste de bonjour.

Fait bravo.

14 1/3. Fait au commandement : bravo, bonjour, au revoir, avec la"main.

Se couche au commandement et de même essaie de se lever. Montre son pied (en le levant), sa bouche (en y mettant le doigt), sa main (en faisant bravo).

14 3/4. Sur demande: "montre ton pied", montre la pantoufle quittée (un jour suivant, montre son pied).

Paraît se tourner sur demande vers sa mère et sa sœur.

Montre son nez.

Sur demande, se porte bouche et bras en avant pour embrasser.

15. Montre sur demande la serviette, la fourchette et d'autres objets.

20. Marche réalisée.

22. Fait semblant de téléphoner (en disant alo).

J. 11 1/2. "Bravo " avec les mains, et les pieds; "vive... " en levant les bras; " bonjour " ou " au revoir " avec la main.

"Coucou " en se cachant avec sa serviette ou son drap.

Fait " câlin " en appuyant son front à la figure d'une personne.

13. Bruit de baiser sur commande (et quand on parle de baisers).

Fait "les marionnettes " (rotation de la main).

14 1/3. Quelques déplacements sur le derrière.

Sait mettre un objet dans un autre.

Dit merci avec le bras au commandement (quelquefois remplacé par $h$ de plaisir).

Bruit de baiser (envoie un baiser) en même temps que geste de la main. Au commandement ou spontanément.

"Où est le nez?" Avance la figure [et répète nene].

"Où est ton ventre? " Habillé, fait le geste d'enlever sa culotte; nu, se tape sur le ventre des deux mains.

Tend la main à la main tendue; l'agite pour au revoir ou bonjour.

Se cache à l'invite "coucou".

Fait bravo, vive..., les marionnettes (avec un seul bras).

"Fume ": moue; souffle, pas toujours, les doigts près de la bouche. 
Imite des attitudes nouvelles pour lui : le grand-père entre dans la chambre en tenant une œillère sur l'œil; imite, en portant deux doigts formant rond à son œil. Le lendemain matin répète ce geste sur l'interrogation: "Qu'est-ce qu'il fait grand-père? »

15. Joue au téléphone avec un téléphone jouet.

Range ses jouets à mesure qu'on les lui nomme.

17. Marche réalisée.

J. L. 12. Sur commande, dit bonjour ou au revoir en tendant la main (à une personne à portée).

Fait non par geste latéral du bras.

14. Marche réalisée.

18. Me donne un pelit pot; je fais semblant d'y boire; il rit, fait aussi semblant, et donne l'objet à son père.

Les dressages sommairement décrits ici sont inséparables dans l'existence de l'enfant du serinage de la parole qui est contemporain. Ils comportent l'emploi abondant de la parole adulte, comme instrument du dressage, conjointement avec la gesticulation. Ils supposent de la part de l'adulte la confiance que l'enfant comprend les mots, à peu près comme lui. En fait, on peut affirmer grossièrement, en attendant des études beaucoup plus poussées par les moyens actuels - et par les moyens futurs - de la psychologie qu'il y a du moins et du plus. D'une part, beaucoup de mots doivent être, pour l'enfant comme pour l'animal, des signaux associés au geste à faire, et non à toutes sortes d'idées que ces mots peuvent éveiller, éveilleront plus tard chez le même enfant dont la mémoire les aura mis en réserve. D'autre part l'enfant, comme peut le faire aussi dans une moindre mesure l'animal, est attentif à toutes sortes de circonstances accessoires que l'adulte peut ne pas remarquer au même moment. En particulier, ce qui ne se produit pas pour l'animal, l'intelligence en croissance de l'enfant peut accrocher diverses choses dans les propos échangés entre les adultes qui procèdent à son dressage.

\section{C. - Acquisition et exercice du langage enfantin.}

En ce qui concerne le langage parlé, la période considérée ici va des premiers exercices de reproduction du langage parlé des adultes jusqu'au moment où la grammaire de ce langage commence à être utilisée.

Mais cette période doit elle-même être divisée en tenant compte de différentes étapes, dont les limites ne peuvent guère être tracées exactement. D'autre part, sensiblement plus que dans le langage total des adultes, des éléments autres que les mots proprement dits entrent en jeu et doivent être considérés de près si on veut comprendre vraiment l'exercice de l'intellectualité et de son instrument de communication à l'âge considéré. 
Il est nécessaire de discerner beaucoup d'éléments et d'opérations sans oublier qu'ils se trouvent à chaque instant entrcmêlés dans la pratique; l'exposé devra donc être divisé lui-même en paragraphes. Mais certaines considérations dominent l'ensemble et doivent être énoncées d'abord.

D'une part, l'enfant répond par l'imitation, ou plutôt par l'effort d'imitation, au dressage auquel l'adulte procède, tant pour l'acquisition des sons et de leur assemblage que pour la connaissance des sens qui sont attachés à ces assemblages dans la langue qu'il est question d'apprendre.

D'autre part, l'enfant perçoit plus ou moins bien, imite en partic mécaniquement et emmagasine dans sa mémoire beaucoup plus que ce qui lui est volontairement inculqué.

D'un autre côté, il n'est pas qu'imitateur : pour répondre à des besoins urgents auxquels il ne peut pas parer par des moyens qu'il n'a pas encore appris, il recourt assez souvent à des créations - qui s'imposent pour leur plus grande partie à la compréhension et à l'acceptation des adultes (une partie demeurant inefficace faute de compréhension (1).)

La création ne s'arrête pas à la première enfance, elle prend seulement d'autres formes, après la normalisation approximative du phonétisme. Dans l'enfance, et plus tard, tenir compte d'usages fantaisistes de "bruits de bouche ". D'autre part une invention plus ou moins active s'exerce chez l'adulte dans la création de mots (en particulier de sobriquets donnés aux enfants et à d'autres personnes), quelquefois d'élćments grammaticaux (formations analogiques de conjugaisons, extensions de suffixes de dérivation, etc.) et particulièrement dans l'agencement suivi du discours (divers éléments du style personnel).

Si nous séparons pour une part dans l'étude et dans l'exposé différentes parties du matériel de la langue, il ne faut pas oublier les parallélismes de développement : pendant la période où l'enfant n'a acquis qu'une portion du stock de phonèmes, il est limité aussi pour la réalisation des combinaisons de ces phonèmes en équivalents enfantins du mot; pour la possession simultanée de beaucoup de ces équivalents de mots; enfin pour leurs agencements en ensembles. A chaque stade, il y a une structure, où les différents éléments s'intercommandent. D'autre part, les stades se compénètrent, par la persévération d'éléments d'abord acquis et par l'apparition de quelques éléments en avantgarde, souvent de manière sporadique; il faut compter aussi avec certaines régressions possibles. Ces phénomènes sont d'ailleurs normaux dans l'évolution des langages adultes et apparaissent quand on les considère historiquement.

(1) Sur l'invention chez l'enfant, voir, outre différents passages de l'ouvrage do Werner F. Leopold cité page 183, l'article du même auteur : a Original invention in infant language * dans Symposium (Syracuse University), III, 1, mai 1949. 
1. Les phonèmes (pris isolément). - Le jeune doit acquérir petit à petit par imitation l'ensemble du stock des articulations qui servent à former des mots (ou "phonèmes") dans la langue ou les langues qu'il apprend.

Il s'agit d'activités et de positions de petits organes (cordes vocales, langue, voile du palais et leur entourage) mus par de très petits muscles, donc de micro-mouvements difficiles à régler et pour lesquels dans l'ensemble la vue donne peu d'indications. Malgré l'exercice préalable du gazouillis, où d'ailleurs toutes les articulations sont loin de se présenter, la tâche est très difficile. On peut considérer que dans l'ensemble l'enfant arrive toujours à la fin de la période d'acquisition prégrammaticale sans posséder et employer à coup sûr tous les phonèmes.

Il en faut un minimum, de manière à former des termes non homonymes, sans quoi il n'y aurait pas de compréhension possible. Donc, malgré certaines homonymies éventuellement tolérées, malgré l'abondance des sens connexes (dans l'usage de l'enfant) qui peuvent être donnés à un seul terme, l'enfant ne peut être considéré comme parleurdébutant que lorsqu'il s'est établi dans son langage un certain nombre de phonèmes contrastant les uns avec les autres, constituant ce qu'on appelle des oppositions phonologiques.

Rappelons que le français, considéré dans la réalité de sa prononciation (et non dans son orthographe), a 35 phonèmes, 20 consonnes et semi-voyelles et 15 voyelles. Les phonèmes se révèlent plus ou moins difficiles à bien réaliser; une partie notable des jeunes Français, fort capables de volubilité dans le bavardage, arrivent à l'âge scolaire sans être capables de bien prononcer les chuintantes (sourde $\breve{s}$, ch de l'orthographe, sonore $z, j$ ou ge de l'orthographe), par opposition aux sifflantes : sourde $s$, sonore $z$. Les consonnes momentanément "difficiles" peuvent être uu remplacées par des consonnes plus "faciles", ainsi $t$ pour $k$ ce qui risque de multiplier lẹs homophonies, ou être articulées imparfaitement, ainsi $s$ mouillé $\left[s^{\prime}\right]$ pour $\breve{s}$, ou être omises, constituant des cases vides dans le tableau des phonèmes et des " consonnes zéro" dans les mots; ceci est surtout fréquent pour les liquides $r$ et $l$.

Les emplacements respectifs des consonnes et voyelles et des consonnes entre elles ne sont pas indifférents : une consonne peut exister seulement à l'initiale (début du mot) et ne pas être articulée en finale, ou ne pas se combiner avec toutes les autres, ou certaines autres, en groupe. Ainsi tel enfant prononce correctement rond, mais grand-père sans $r$ [g $\left.\tilde{u} p_{\ell}\right]$.

Un grand nombre d'enfants devront être observés, en tous pays, de manière à reconnaître les règles d'acquisition. Dès maintenant, il faut savoir que c'est un des points où se manifestent nettement les différences individuelles.

Pour juger du phonétisme d'un enfant et de son acquisition, il faut naturellement tenir compte de son entourage, et ne pas lui attribuer 
une originalité ou une faiblesse qui n'est qu'une imitation. (Voir encore ci-dessous sous 2 .)

Il n'est pas donné ici d'étude de l'acquisition du phonétisme pour les sujets observés (1). Les lecteurs pourront extraire eux-mêmes certains renseignements des notations données dans les divisions suivantes. Voici cependant à titre d'exemples quelques indications sur J. et J.-L.

Ces deux cousins, de même âge à un mois près, élevés à Paris dans des conditions peu différentes, ont présenté un contraste net et instructif pour les moments d'acquisition des phonèmes.

J. Quelques notes de la période 18-20. Distingue très bien entre eux $p$. $t, k$, avec lesquels $b, d, g$ sont généralement confondus ( $b$ dans byẽ, voir p. 211) : donc trois séries d'occlusives, mais sonores confondues avec les sourdes.

Emploie les nasales $m, n$ et une sorte de $\tilde{n}(g n)$.

Emploie un $l$ net en diverses positions; $r$ est généralement absent.

Les spirantes dentales (sifflantes) et prépalatales (chuintantes), ainsi que les labio-dentales manquent. Exemples $a$ "chat " $\sigma$ (on) " garçon " et "bouchon " iñ " cygne ", $\ddot{o}(e u)$ et lö " fleur ", $w a$ " voir ", yo " avion".

[ $f$ et $v$ sont notés à $21, s, s, z$ et $r$ commencent à 22].

J. L. Relevé fait par son père, à 19 et 7 jours.

Distingue $p t ; k$ est généralement remplacé par $t, k$ est exceptionnel. Les sonores $b$ et $d$ en général confondues avec $p$ et $t$; l'articulation sonore est exceptionnelle; $g$ est noté comme absent, ainsi asõ " garçon 》.

Les nasales $m$ et $n$ sont employées; $l$ est totalement absent (prononciation zéro) ainsi $₫$ que $r$.

La siflante sourde $s$ est bien établie (mais prononcée un peu zozotée) $z$ semble absent : mẽ " maison "; $s$ est établi, bien distinct de $s$, un peu mouillé $\xi^{\prime} a$ " chat ", $z$ est absent : " Jean-Louis " se réduit à $a-i$.

Le / est bien établi (mais bi labial); $\varphi$ est absent.

[ $l$ apparaît sporadiquement à 22 , mais une somme faite à 24 note : absence de $k, \breve{s}, l, r ; \dot{a} 25, r$ commence à s'établir, à $26, r$ et $l$ sont notés comme généralement employés; $k$ et $g$ semblent avoir commencé à s'employer].

Des observations piquantes sur J. montrent comment deux ou plusieurs stades d'acquisition peuvent être représentés dans une même étape d'ensemble, c'est-à-dire à la même date, dans des mots différents, acquis à des moments différents ou dans des conditions différentes, ou présentant des figures phonétiques particulières.

A $191 / 2-20$ il a présenté trois traitements de $m$ initial. $m$ dans $m \tilde{a} m \tilde{a}$ (mot à répétition tout entier nasal) qui était acquis à 17 . zéro, dans ayi "merci ", $a i$ "Marie ".

$p$ dans pal o pye " mal au pied" (favorisé par le $p$ suivant?).

A 21, $p$ est introduit dans payi "Marie".

A 23, le nom est rectifié (ou rappris) sous la forme mai.

(1) Se reporter aux études de Leopold (vol. I et surtout vol. II) et de Grégoire. 
(Note de mai 1952). - $\Lambda$ l'âge du langage acquis et de la conversation courante, des différences subsistent entre l'articulation des deux sujets. A 2 ans 9 mois, J. a une articulation normale où cependànt les sourdes ressemblent encore quelquefois à des sonores (ce qui n'est plus

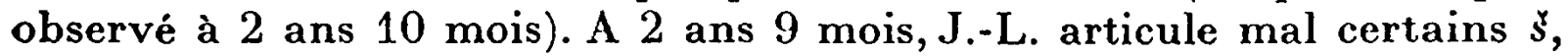
prononce souvent $z$ pour $\dot{z}$ (dont il a pourtant présenté des exemples de bonne heure), il remplace les palatales $k$ et $g$ par les dentales $t$ et $d$.

2. La figure des éléments correspondant aux mots du langage adulte (1). - Il est vain de faire seulement le tableau des articulations isolées; dès le début de l'observation il faut considérer leurs groupements.

Le fait général est que ces éléments parlés de la première période sont dans l'ensemble constitués d'une seule syllabe au premier stade du langage enfantin. Les mots de la langue se trouvent donc réduits, sauf ceux qui sont très courts et qui prennent paradoxalement dans la langue enfantine l'allure de privilégiés.

Les causes de réduction sont convergentes : la principale est sans doute une incapacité du jeune à cette étapc de croissance de manier un assemblage autre que très simple de ces éléments qu'il a encore de la peine à articuler. La syllabe qui est scule conservée et tient lieu du tout est en français la syllabe finale; c'est celle qui porte un accent léger mais suffisamment net.

Le monosyllabe peut avoir des formes déjà assez variées pour que leur emploi avec les divers phonèmes acquis permette la constitution d'un vocabulaire différencié (ainsi les théoriques $p a, a p, p a p, p l a[(p) a l p$ ou (p)apl devant être considérés comme tout à fait exceptionnels], et de même ta, at, tat, tra, etc.).

A côté du monosyllabe, le terme du type dodo à deux syllabes identiques figure quelquefois dès la première période, soit fourni par les adultes parlant plus ou moins "bébé ", soit créé par les enfants par accommodation d'un mot (rarement d'un mot avec article). Il n'est pas sûr que des éléments à deux syllabes non pareilles ne soient pas normaux encore que rares au même stade.

Lors de la croissance de l'enfant et du vocabulaire, les longueurs des éléments parlés augmentent, en même temps que le nombre des phonèmes et de leurs combinaisons, et celui des termes eux-mêmes.

Dès qu'on sort de l'émission à une syllabe, les questions de l'harmonisation ou de la différenciation des syllabes se posent, ainsi que celle de la place et de la nature de l'accent tonique. Ici, comme pour les articulations isolées, il faut tenir compte de l'entourage. D'une manière générale et naturelle, il se trouve que les adultes parlent plus ou moins "comme leur mère", soit restée dans son milieu, soit transplantée; ainsi un petit Parisien peut montrer des traces plus ou moins nettes 
de l'accent d'une mère méridionale. Mais le père, un des grands-parents, une personne quelconque prenant soin de l'enfant la plus grande partie ou au moins une partie notable de la journée, peut être en question. Un exemple, qui est dissimulé dans la suite de l'exposé par l'uniformisation de la notation mamã pour "maman", se rencontre chez J. : à côté de cette prononciation, qui est celle de sa mère, it emploie aussi et semble-t-il de préférence la forme mama avec un seul $a$ nasal; or c'est la prononciation employée par son père, qui lui parle beaucoup.

Des études nombreuses devraient être faites avec essai pour discerner des stades caractéristiques et évaluer leur durée.

Ci-dessous des coupes pour mes cinq sujets, portant sur une journée, à 15,18 et 24 mois (à quelques jours près).

Fr. 15. $m \pi m \pi$, quelquefois $m \pi(m)$, avec $\tilde{a}$ teinté de $e$ " maman ".

pa-pa (détaché ainsi) " papa ".

lla, l'a, la " là, là-bas ".

bam, bam = "boum, pam ».

osi, $2 s^{\prime} i$, sentiment de satisfaction (provient de " merci 》).

$\widetilde{\boldsymbol{e}}$ " hein?" (et divers autres grognements).

sä, $s^{\prime} \ddot{a}$ " ça! ".

täd, de signification inconnue (provenant de ça y est précédemment essayé?). $p a, p \check{c}$ " pipi " (noter la simplification du mot à répétition).

$p a, p e$ (quelquefois chuchoté, phénomène qui s'observe parfois chez les enfants) " pain".

tata (chuchoté) ou toto (marqué d'un ?) "cocote".

te " par terre".

adya "Marianne ".

$18+10$. Langage spontané.

mãm $\tilde{a}$ maman.

papa, papapa papa.

te par terre!

pa-pa pipi.

po du pain!

कō " voilà, c'est bien!».

$a(y)_{\varepsilon}$ " çà y est ".

$a^{\mathbf{T}} \boldsymbol{s} \tilde{a ̈ r}$ " au revoir ".

$s^{\prime}$ (rare) " merci ".

te te (rare) " dodo".

Répète sur proposition :

$p a$ "pain ", $p$ " soupe ", koko " gâteau ", baba, br̃bro " bonbon", mẽ " main ",

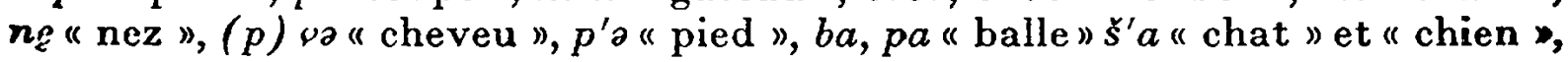
oa " cheval ", fo "feu ", pipi " pipi ", sa "sage " (à peu près) mnyəmmnyom a mignon ", kok? " coucou " et "encore ", am, man, ma " ham!".

24. (Sur le seuil de l'âge grammatical; tout spontané :)

pa de pis "pain d'épice ", aeeus' "tablier rouge ", paş̨l "Marcel ", atür " voiture ", kwa " trois ", tat " quatre ", ola " voilà ", apuf " la soupe ", fuit, wit a frites ", fasis "Francis ", ellela " il est là ", o apęl " il s'appelle ", titatęr " pomme de terre $»$. 
L. 15. « Poule » (proposé) $k^{\mathrm{r}} \dot{a} k^{\mathrm{r}} \dot{a}(\tilde{n})$.

"Papa " (proposé) $k^{\mathrm{r} a} \mathbf{k}^{\mathrm{r}} \dot{a}$ et $\mathrm{papa}(p a)$.

"Fleur $v$ ou souffle fort à lèvres avancées, $f$ ou $h$.

as'an (à peu près) "Christiane " (spontané).

patan patan (à peu près) "canard ".

18. Mots proposés : fü " confiture ", pipi « pipi ", $d u$ (avec $d$ sourd) et tetu " c'est tout ", mämä (ä légèrement nasal) " maman ".

24. Spontané : ali, alli "sali ", adęn " descendre ", uy "grenouille ", pętït " culbute ", pul " poule ", alüm " allume ".

Chr. 15. (Répété), papa (chuchoté), ava "au revoir ».

18. aba, apa "là-bas", pipi " pipi "; la note dit que papa est rare.

24. don " je le donne ", odo " où donc? ", kaka " âne " (origine?); en montrant une chaussure : potapi (origine non déterminée; en tout cas, émission de trois syllabes).

J. 15. [Lettre de sa mère] néné en montrant son nez, jouah " joie ", décaca = "délicat" entendu dans la conversation" (trois syllabes ensemble).

18 1/2 Spontanés : bum, pum "poum! " bum-bum "pan ".

am " mange! ".

ala " le voilà ".

byi" " bien!".

tun "tourne, tourner".

töy "fauteuil ".

batũ « bâton ".

$u$ " un chien aboie".

pati-tan "Christiane est partie".

kis-tan "Christiane " (sur proposition).

$231 / 2$. (Au seuil de l'âge grammatical).

Spontané : mốt $\tilde{r}_{e}$ " montrer ", grüme "grand-mère ", fizęl " ficelle ", ak ka " encore ", pøy a papye "corbeille à papier", mamã et mäma "maman", ayu, kayu "caillou ", dïpé "du pain ". Répété ter "secrétaire ", manan * banane ", slö «fleur».

J.-L. 14 1/2. [Lettre de sa mère] ppa-pa "papa? " surtout quand il est seul; papo quand on lui parle de chapeau, ssis "Francis" (quand on en parle).

18. müzilk " musique "(T. S. F.), $k^{\prime} a k^{\prime} a, a k^{\prime} a$ (? dit en montrant des fleurs), kato " gâteau " (pourtant $k$ est $t$ encore plus tard; voir ci-dessus), po-po " un

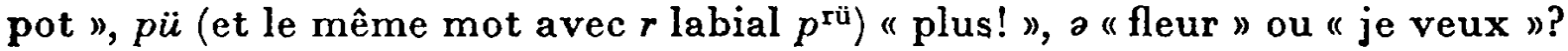
$p \varepsilon$ " grand-père ".

24. ãpe " grand-père ", ñ̃ " non ", o " encore ", pipi " pipi ", mãmã " maman ",

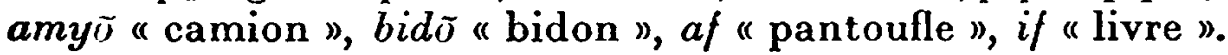

On voit que dans les documents ci-dessus le rôle des termes à répétition est plutôt réduit.

Pour des interprétations valables sur le mode de croissance des termes parlés (allongements et rapprochement de la correction) il faudrait naturellement des coupes plus "épaisses", portant sur des périodes 
bien découpées suivant les faits eux-mêmes - on ne peut pas indiquer une mesure fixe - et très abondantes en notations.

Il faut naturellement distinguer entre les termes adoptés dans les premiers temps et ceux qui s'ajoutent successivement, à des moments où le phonétisme est plus riche en phonèmes et en assemblages. Pour chacun des termes qui se rectifient, la question se pose de savoir si la forme ancienne est corrigée progressivement, ou s'il se produit une substitution d'une nouvelle forme apprise. La réponse n'est pas douteuse quand il y a changement de terme, par exemple si wousyou cède à chien. Mais dans les autres cas l'interprétation est généralement très délicate.

Il faudrait pouvoir explorer ce qui se passe dans la mémoire, dans les perceptions et dans la conscience linguistique de l'enfant. Dans quelle mesure la forme qu'il emploie se substitue-t-clle à celle qu'il a entendu, et qu'il réentend plus ou moins souvent? Dans quelle mesure a-t-il conscience qu'il n'imite pas bien et s'efforce-t-il de faire mieux, en se référant à ses nouvelles acquisitions (1)?

3. Le serinage.- Il convient, pour apprécier les vitesses de croissance des langages, de noter soigneusement comment se fait l'apprentissage, dans quelle mesure il est mené et par qui, à quels moments il y a proposition, proposition répétée, proposition détaillée par syllabes, rectification de ce qui a été dit par le petit, interrogation sur le nom des choses.

Des termes peuvent avoir au même moment des formes différentes dans l'emploi spontané et dans l'emploi provoqué; mais il y a aussi des variations à l'intérieur de l'un et de l'autre de ces emplois.

D'une manière générale, les parents ou autres personnes de l'entourage de l'enfant lui donnent un certain enseignement du vocabulaire, en présentant dos mots isolés.

Il peut arriver, même dès un âge tendre, que l'enfant provoque ou semble provoquer cet enseignement, en avance sur les questions que tous posent à des stades ultérieurs.

C'est le serinage qui donne aux enfants le répertoire d'imitations de bruits dont ils ne connaissent qu'une partie au plus par expérience personnelle : "Comment font les chiens, les chats, les moutons, etc.? "

Dès cette période aussi il arrive que les parents ou l'entourage enseignent aux enfants des chansons ou même des fables. L'enfant commence par ne répéter que des syllabes de rimes ou quelques mots qui l'accrochent. Il peut parvenir à tout apprendre, même les airs s'il a des dispositions musicales. En général, dans ces textes, tous les sens de phrases et la plupart des sens de mots lui échappent. Néanmoins il peut arriver que certains mots courants s'implantent ou se stabilisent par cette voie.

Noter que la mise en mémoire précoce de textes suivis ne fait qu'anti-

(1) Voir Langages successifs, p. 113-116. 
ciper sur des récitations scolaires de textes littéraires mal compris et sur des lectures plus ou moins mécaniques, surtout chez des bilingues dont la "grande langue " n'est pas la langue maternelle. On peut mentionner ici aussi l'usage, fort répandu dans le monde, de langues religieuses non comprises, latin chez les catholiques, arabe écrit chez les Musulmans, etc.

Pour ce qui est de l'emploi des mots et du mélange avec d'autres moyens de communication dont il va etre question ensuite, il y a pratiquement initiative de l'enfant soit dans l'imitation même (ce qui n'est pas une contradiction, puisque l'imitation s'exerce avec plus ou moins de choix et de stylisation), soit dans une création, qui elle-même peut n'ètre pas exempte d'éléments d'imitation difficiles à déceler.

4. L'emploi du langage. - Dans le terme langage est compris ici pour l'enfant, comme plus haut pour l'adulte, tout ce qui sert à s'exprimer volontairement, en extériorisant une volonté, une constatation, un récit du passé, une anticipation, une fiction, des sentiments variés, de manière compréhensible ou au moins voulue telle, à l'adresse de l'interlocuteur ou des interlocuteurs.

L'essentiel est la communication, le moyen employé est secondaire. Il est vrai que la parole domine nettement les autres moyens, assez tôt chez le jeune enfant et de plus en plus complètement par la suite et que, sous forme muette, elle est seule en question dans le langage intérieur normal. Mais on ne doit pas pour autant considérer les débuts du langage chez l'individu de la même manière que le langage développé, sinon on risque de très mal comprendre.

Aussi se pose-t-il une question de nomenclature. Pour éliminer les confusions, nous devons éviter d'employer celle qui s'applique au langage adulte, donc laisser de côté les termes "mots" et "phrases". Par quoi les remplacer? On pourrait dire : élément linguistique ou élément de communication. C'est long; peut-être peut-on risquer une abréviation : élémcom? Pour les emplois combinés de deux ou plusieurs de ces éléments, il suffit de dire "assemblage".

Une fois ces points fixés, reportons-nous à ce que nous avons dit sur les différents moyens de communication de l'adulte : c'est maintenant le moment de dire que tous ces moyens se rencontrent chez l'enfant, avec des proportions et en partie des emplois différents.

Dans ce qui suit seront donc mis sur le même plan, comme des élemcoms divers : le geste (qu'on pourra appeler gestcom), les cris, grognements, gémissements, etc. (qu'on pourrait réunir sous le nom de gémcom) et les représentations de mots du langage adulte, qui seront des élémcoms parlés (ou plus courtement parlcom) (1).

(1) Leopold affirme plus d'une fois la nécessité de ne pas juger le fonctionnement du langago enfantin commo celui des adultes (voir entre autres $I$, p. 166-177; III., tout entier, avec la plus grande densité d'indications au début); pour la nomenclature, il a pris des demi-mesures; des 
Ici se présente une question souvent débattue, qui a été posée de manières incomplètes et fausses, sur des observations insuffisantes, en partie interprétées à faux à cause d'idées préconçues et de la méconnaissance de facteurs importants.

Il s'agit de savoir quelle est la part de ce qui est affectif, émotif, et de ce qui ne l'est pas.

Il s'est trouvé que sensiblement au moment où des études de stylistique ont montré qu'on avait trop négligé la part du sentiment dans le langage parlé et aussi écrit, on a été porté à trop diminuer la part de ce qui n'est pas volonté exprimée dans le langage enfantin et on a interprété comme des ordres ou des désirs des éléments de communication (élémcoms) qui sont des constatations ou des récits : c'était une erreur.

Mais s'il faut rétablir dans leur rôle principal ces éléments froids et relativement statiques, on doit aussi reconnaitre ce qu'ils comportent de rôle actif.

Quand l'enfant se livre à des constatations du type de boum (= la chose est tombée), il est probable qu'il s'exerce à la fois à penser et à parler, à un moment de son existence où on peut croire que le langage intérieur ne fonctionne pas ou ne fonctionne pas à plein : il y a donc dans l'émission de ces sons une activité utile, nécessaire pour la croissance et pour les actions futures. (Pour ce qui est du langage, l'emploi répété affermit les articulations et aussi les identifications de sens).

Du même coup, l'enfant accomplit encore un autre genre d'exercice : il s'exerce à la sociabilité. A cet effet il emploie précisément les moyens de communication. Ceux-ci donc, encore sous cet angle, sont actifs, même s'ils sont d'expression statique.

Et maintenant voici un choix d'observations, avec quelques considérations complémentaires. Indépendamment des densités différentes de notes prises, il faut tenir compte des inégalités de croissance chez les enfants; mais on ne pourrait bien juger qu'en tenant compte à chaque instant des données qui leur ont été fournies par l'entourage.

a) Apparition et caractère des premiers éléments de communication. Nous allons voir ici dans leur pêle-mêle les premières manifestations qui ont été relevées chez les sujets d'observation.

On peut se demander si les a ou ä qu'émettent les enfants à partir de 2 mois n'ont pas quelquefois un caractère de communication, étant

gémcoms apparaissent comme interjections et non-standard words, des parlcoms sont *words * (entro guillemets) les assemblages sont souvent des non-standard sentences; dans III, p. 8, apparaît lo terme activity pour désigner ce qui dans une vraio phrase serait nommé verbe, même s'il s'agit de termes qui ne proviennent pas de verbes du langage adulte. Quant aux gestes, il déclare * que ce ne sont pas des éléments linguistiques * (III, p. 158 bas). 
provoqués par une présence. Mais c'est douteux. (Voir ci-dessus p. 197 et ci-dessous Fr.) (1).

Fr. Avant 9 mois, on n'a noté que des genres de gazouillis ou des réponses à des propositions.

Voici ce que j'ai noté à 9 mois : sur proposition " attends, attends " répond atä ou analogues, ou bien fait non de la tête, en semblant refuser de répondre.

A la même date : comprend le non parlé ou le geste de la tête employé comme défense, et y obéit quelquefois. L'équivalence des deux élémcoms pour lui semble prouvée par le fait que sur l'invite non destinée à le faire énoncer éventuellement la même émission il répond en faisant le geste.

D'autre part : "exprime spontanément le refus par un grognement ".

Enfin, voici pour la sociabilité ce que j'écrivais à cette date :

« La conversation (spontanée) ne comporte pas une véritable articulation. C'est une émission de voix, avec l'appareil articulatoire relâché, qui sonne généralement à peu près $\ddot{a}$, souvent $\partial$; pour peu que la bouche se ferme, on entend $\tilde{e}$. L'émission est plus ou moins prolongée et nuancée et accompagnée de mines. $n$

$9+8$. Fr. exprime spontanément la demande (sans qu'on lui ait jamais appris) en tendant les mains et les ouvrant et fermant plusieurs fois.

$9+17$. Fr. commence à montrer spontanément à des personnes voisines des objets qu'il tient dans la main.

$10+3$. Fréquemment äyä, exclamation de tendresse ou espèce d'énonciation; notation analogue a $121 / 2$, on peut se demander si ce terme ne provient pas de " ça y est ".

$10+13$. Ne fait plus non de la tête.

$121 / 2$. Un élément court ye a la valeur " oui ».

14. Dit « là ".

15. Voir ci-dessus, p. 210.

La note du jour ajoute : peu de gestes-langage; montre en disant ld [geste d'accompagnement, qui n'a pas été noté antérieurement comme gestcom autonome]; tend les bras pour être pris ou mis debout.

$151 / 2$. Le terme le plus employé est toujours $l a$ " là "; $\tilde{e}$. est modulé différemment pour l'interrogation, l'affirmation, etc. A non de la tête s'est ajouté oui de la tête. Le bras agité latéralement veut dire " sale! ».

L. $9+20$. Note peu claire : a comme conversation, et aussi des atätä et analogues.

$10+23$. Semble dire " non " avec la tête.

11. Note reproduite textuellement. "Emploie de plus en plus une espèce de souffle (admiration?), semble faire ho quand quelque chose tombe; semble demander quelquefois à aller sur le pot. Sa mère dit : " Elle va parler ".

11 1/2. Ne dit plus non de la tête.

(1) Je laisse le texte tel qu'il a été écrit en septembre 1951. Mais j'ajoute ici les premières observations sur un nouveau sujet, Yves, frère cadet de J. L., né le 30 juillet 1951 . Les sourires auraient apparu à 1 1/4. A 1.20, il émet ə-ə tranquillement, en attendant de boire. A 2. l'émission $2-\partial$ serait devenue une conversation, d'après l'observation des parents. Voici ce que j'ai observé moi-même, à 4.25 : l'enfant couché sur le dos fait des modulations vocaliques à pleinø voix; je m'approche de manière qu'il me voie; les modulations variées s'interrompent; plusieurs - cont émis, assez vigoureux et un peu modulés; j'ai eu l'impression nette d'une communication. 
$113 / 4$. Semble dire $n e, n e \tilde{e}$ en faisant non de la tête; en général le refus est exprimé par un grognement caractéristique.

$12+4$. Une note dit : premiers mots nets : la bouche en position de sifflement et émission d'un léger son, "c'est joli "; la bouche en position de souffle, léger $p(u)$, "c'est tombé ".

$121 / 4$. Non de la tête ou grognement.

Oui de la tête, quelquefois.

Bras tendu vers une personne pour l'appeler.

$121 / 2$. Une note dit : "Semble dire beaucoup de choses que nous ne comprenons pas; dans la variété de ses exercices il semble y avoir des mots."

Fait " merci " du bras; spontanément?

13. A un grognement impératif ên très caractéristique, pour demander des objets, notamment à manger.

$13+6$. On note que manemane employé comme exercice, avec une nuance d'impatience, est employé quelquefois isolément (notamment avec la main tendue) avec apparence de demande.

Confirmé à $131 / 2$ comme "réclamation ".

13 1/2. [Voir ci-dessus p. 198.] La note dit : aucun mot net (entre autres : ta " tiens "?).

Deux jours après : " $t a$ tend à se confirmer, correspondant à tiens, voyons, donne; quelquefois tya.

14. Augmentation des phrases incompréhensibles; beaucoup de mots doivent nous échapper.

14 1/2. Note de bilan : aucun mot net, sauf non, pas toujours employé. Mots indistincts pour "chapeau ", "Christiane ".

Pas d'usage de papa et maman.

Un o chuchoté veut dire : "c'est tombé 》 ou "c'est vilain 》.

14 3/4. "Toute communication de sa part sur le ton de conversation est $\ddot{a} y^{\prime \prime}$, avec le doigt tendu, généralement, ainsi pour chapeau à la patère, images au mur, etc. "

Désigne spontanément des aboiements de chiens par quelque chose comme $\overline{\tilde{o}}, \ddot{o}(1)$.

(Par ailleurs, pour la même date, voir division suivante.)

A cette date, et peut-être antérieurement : ronronne à peu près comme un chat, quand elle mange avec satisfaction (mais non en suçant des bonbons).

15. "Ses mots deviennent beaucoup plus variés sans être encore comprẻhensibles. "

Le lendemain on note que $\bar{o} \bar{o}$ s'applique au train comme aux chiens; le surlendemain s'applique au chauffe-bain.

En même temps, note rétrospective : " Depuis longtemps geste spontané de demande par mains ouvertes et fermées. "

$151 / 2$. En voyant son pot, dit po : cette constatation est le premier mot net pris au langage adulte.

Chr. $81 / 2 . a$ sert à appeler.

10. Pour oui, semble employer un secouement de tête analogue à celui de la négation, et $\tilde{e}$.

(1) Langages successifs, p. 114. 
13. Je note que depuis longtemps les expressions de " non $»$ et " oui » ont disparu.

14. Tend la main pour dire bonjour?

15. mamama est une demande; quelque chose comme apé est un contentement-remerciement.

15 1/2. mama "maman " serait quelquefois employé avec son sens.

$153 / 4$. papa est noté comme employé.

J. Une note à 10 , signale une "conversation " au lit avec yä, gya et des ata, ada accompagnés de battements de bras : pas délémcom net.

$112 / 3$. tö est une " conversation "; aaa exprime la joie; $h$ ou $a$ avec bouche très ouverte veut dire "que c'est beau! ".

Tend la main pour dire bonjour.

Un grognement pour demander la nourriture.

Fait non de la tête.

12. L'émission $t \bar{a}-t a$ qui à $112 / 3$ répondait à la proposition "papa ", est employée pour désigner son père et pour souligner le retour à la maison après promenade.

13. Continue à dire non de la tête.

Il semble que le bruit de baiser, employé sur commande et quand il entend parler de baisers, serait aussi un signe spontané de contentement; celui-ci s'exprime d'autre part par àtä.

(Notes de bilan pour une petite période.)

$141 / 3$. $h$ léger et prolongé, à bouche grand ouverte continue à vouloir dire " c'est beau, c'est bon " et " merci ». $a-\bar{a}$ plus ou moins crié : " à manger! * ou " à boire! ".

De plus $f$ émis en jouant avec une fleur a peut-être aussi le sens $\alpha$ c'est joli » à moins que ce ne soit un abrégé de fleur.

Fait non et oui de la tête.

Le tã, ta précédent semble servir de communication vague, et aussi d'accueil?

Il semble que baba, papa, mama commencent à s'employer indifféremment pour père, mère et aussi grands-parents.

Un cri prolongé, à constriction laryngale et sur tonalité de $i$, aurait d'abord été un cri de plaisir en touchant des cheveux; il est employé statiquement quand il s'agit de cheveux (invité à montrer sa tête, à mettre un objet dessus, subissant le lavage de tête, entendant parler de cheveux).

$141 / 2$. Une personne étant sortie de la chambre, il fait spontanément au revoir du bras; sa mère interprète : au revoir retardé (par timidité?); je me demande si ce n'est pas un récit : "la personne est partie ".

S'adresse à un objet tombé : $b a, b a$.

ao "tante Christiane " (à qui on téléphone souvent).

17 1/2. Emploie papa, mamã (ou mamã), tan aChristiane (sa tante), assa " au revoir » (spontané?).

18. (Témoignage de sa mère.) Ronronne souvent en mangeant, surtout le dessert ou le pain.

Dit $t i t \tilde{~}$ en touchant des choses soyeuses (proviendrait de " dis donc, coquin ${ }_{\text {, }}$ dit par sa mère alors qu'il touchait ses cheveux ou ses bas). 
J.-L. 10. Quand on dit « tu viens », répond en tendant les bras.

12. Arrivant chez nous, me regarde et me dit $\ddot{o}$; quel sens?

Demande, en émettant $i, i$ " je veux manger » ou " boire ».

Exprime sa satisfaction du manger par (approximativement) myam myam. Refuse par mouvement latéral du bras.

$141 / 2$. D'après une lettre de sa mère " s'exprime surtout en gestes impératifs ".

18. [Voir ci-dessus p. 211.] Ajouter : à la demande "tu dis bonjour? » fait un bruit de baiser et tend la main; fait un geste (quel?) pour demander a sortir de la pièce.

Émet tututu sur un ton de récit.

En somme, très grossièrement il est justifié d'envisager une période où les enfants commencent à disposer de quelques élémcoms de nature variée (gestcom, gémcom, parlcom), au nombre de 10-20 pour fixer sommairement les idées, pendant quelques mois, peut-être en moyenne 6 mois. Les circonstances, apparemment beaucoup plus que les tempéraments des enfants et les intentions des parents, constituent les stocks variés de ces premières manifestations. (On peut constater le role restreint de " maman " et " papa ".)

b) Eléments de communication en abondance, employés isolément. A partir d'un certain moment variable, qui constitue le début d'une nouvelle phase dans l'acquisition, les éléments de communication se mettent à foisonner (jusqu'à un nombre de l'ordre de 200), et les éléments parlés (parlcom) prennent peu à peu la prépondérance qu'ils garderont ensuite.

Dans toute la première partie de cette période les éléments sont employés isolément, les assemblages s'il s'en produit sont exceptionnels.

Or, il existe une répartition intéressante des élémcoms. Beaucoup de parlcoms, et quelques gestcoms, servent surtout à des constatations et à des récits, qui sont beaucoup plus précoces qu'on ne le dit généralement.

Par contraste, les expressions des émotions, des acquiescements et refus, des désirs, paraissent être le plus souvent des gémcoms (grognements, cris) ou des gestcoms qui ne servent pas à la constatation. ici.

Quelques exemples sont donnés sous $a$; il y en a beaucoup d'autres

Cependant bien des parlcoms peuvent exprimer des volontés.

A partir d'un certain moment apparaissent des parlcoms spécialisés dans la volition, comme les formules polies "s'il te plaît " ou équivalent (voir le $v \ddot{o}$ tardif de L.).

D'autres encore apparaissent dans ce rôle lorsque se multiplient

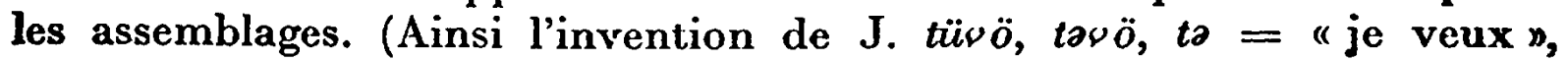
qui est employé en premier élément d'assemblage à partir de 21 2/3.)

Les assemblages, à partir d'un certain moment, se multiplient alors 
que l'usage des éléments isolés persiste, ce qui fait qu'il y a un large chevauchement entre cette division $b$ et la division $c$ qui suit.

Ici une remarque qui vaut pour l'ensemble des observations sur les parlcoms. Il ne semble pas niable que les mots isolés reproduits comme parlcoms par les enfants sont souvent abstraits par eux des phrases des adultes, et il est légitime de se demander par quel mécanisme la chose se produit et comment certains mots ont obtenu un relief particulier quelconque qui a favorisé leur isolement. Mais il faut tenir compte aussi des coupes effectuées par les adultes eux-mêmes. Ainsi "tu veux venir avec maman" peut être occasionnellement découpé par la mère elle-même s'adressant à l'enfant: a tu veux [ou tu ne veux pas]? "; "venir avec moi? pas venir? "; " avec maman?".

Il convient d'observer spécialement l'interrogation, qui semble apparaître à des périodes très différentès suivant les individus. Il faut naturellement distinguer à quoi elle s'applique. A côté des interrogations, il y a les réponses nettement distinctes des élémcoms spontanés.

On pourrait discuter pour savoir comment désigner les appréciations "c'est beau, bon " ou au contraire "c'est sale"; dans quelle mesure émotion ou constatation?

Les termes " constatation, description, assertion " sont plus ou moins malcommodes; l'anglais "statement " est plus maniable. Dès qu'il s'agit du passé, "récit " peut être employé même quand il s'agit d'un élémcom isolé. Une "prévision " est rare. Mais d'une manière générale la notion qu'il faut introduire et utiliser est celle-ci : il s'agit d'eléments de conversation.

L'enfant a de nombreux moments vacants, sans besoins matériels à satisfaire immédiatement, où il se trouve avec les grandes personnes sans leur demander constamment des objets, des jeux ou des caresses, mais où, avec ses moyens rudimentaires, il converse avec elles.

Fr. 16. Répond non de la tête et oui de la tête avec une petite émission de voix.

Demande les noms des choses, qu'il touche en disant $l a$.

$181 / 3$. Répond oui ou non de la tête avec un grognement.

Converse en désignant les objets du doigt tendu et en disant $\ddot{o}$ pour affirmer ou interroger.

Le mot mãmã sert à demander, notamment à être pris dans les bras; te sert à demander à être mis par terre; po à demander du pain, koko à demander " encore $n$.

Malheureusement les notes de cette date ne disent pas comment au juste sont employés des mots comme " chien ", "cheval ", " main ", " sage ", etc.

$191 / 4 . b \tilde{b} b \tilde{\sigma}$ " bonbon " est une demande; toto veut dire " je me couche » ou " mon ours est couché ", bam " je suis tombé ", apo " on m'a mis mon chapeau " ou " maman a son chapeau ".

L. Voici quelques notes, assez pauvres.

$15+4$. En entendant un avion, dit ana, puis ayä yä, puis ōō (aboiement). 
Le même jour, voyant un dirigeable, l'appelle $p . p . p$. puis dit des choses incompréhensibles où $p$. $p$. intervient; plus tard $p$ est appliqué autrement (?) notamment pour le biberon (1).

$15+5$. En passant à l'endroit où elle a crié $\bar{o} \bar{o}$ « aboiement, chien », elle répète plusieurs fois $o o:$ il y a donc manifestation de souvenir, et équivalent d'un récit : "Ici il y avait des chiens aboyants l'autre jour. "

$151 / 2$. En voyant paraître l'œuf qu'elle va manger : $p^{\mathrm{b}}=$ œuf ou « manger '? En prenant la coquille vide : o (= ouf?); me la tend vers la bouche en disant $p^{\text {h-pa }} p a p a$ (imitation de l'appréhension par les lèvres?).

En montrant le fond d'un vase vide : papa "c'est là-dedans qu'on fait pipi ", ou analogue.

$16+5$. nọ veut dire "oui "(?); non est généralement un grognement; non $\varphi$ est une demande.

$161 / 2$. nọ veut dire " non " et s'accompagne d'un geste énergique; mämämämä est une demande éplorée.

$163 / 4$. En montrant par la fenêtre, énonce lï puis dï; c'est sans doute "il pleut" ou "il a plu".

18. pleple "s'il te plaît ".

$181 / 4$. Emploie $\bar{e}$ et $\tilde{e}-\tilde{e}$ pour " oui " (usage exact non noté).

21 1/2. Emploie un jour wi " oui ", comme consentement et approbation; pour le lendemain j'ai noté : $\tilde{e}$ puis swi et vi.

$24+20$. Emploi de $v \ddot{o}$ " je la veux ".

$22+2 . w i$ wi avec insistance pour demander, et wi sur un autre ton pour accepter.

$28+11$. "oui " est devenu pi.

[Voir p. 239.]

Chr. 16 1/2. Dit beaucoup de choses (des énonciations) en mots incompréhensibles pas très variés.

17 1/4. manam manam est une demande.

En tapant sur la table : pam.

18. peta et approchants (non compris par nous) sont des « motifs de conversation par le ton $"$.

18 1/2. Une espèce de souffle suivi de $i$ signifie " oui ".

$\tilde{e} \tilde{e} \tilde{e}$ est employé pour la demande.

$\checkmark$ est peut-être employé pour " merci n.

nä suivi d'émission vocalique nasale est " non ".

$183 / 4$. Pour " oui " on note $\ddot{w} i, w i, p \ddot{w} i, f$.

Désigne des objets avec un $a$ interrogatif, semblant demander leur nom.

21. Fait non avec sa tête pour refuser.

J. 14 1/2. Un geste servant de récit? (Voir cette indication douteuse, p. 217).

$19+6$. Demande des choses à sa mère, surtout un peigne dans ses cheveux, en grognements nasaux, intonés, soit deux à la suite, soit plus (séries de 4), chaque unité étant plus ou moins prolongée.

Regarde des tableaux et autres choses en disant : $0, \delta \delta$ " c'est beau! ».

(1) Langages successifs p. 117. 
Montre des objets tombés en disant bum ou pum. (Voir encore ci-dessus p. 211.)

En disant $u$ signale qu'il entend aboyer un chien.

$19+7$. Je note $:$ il constate, ne raconte pas.

Par exemple ka-ka ou $a$ ka "gants ": " on me met mes gants ».

Autres constatations, sans mots nets : $a$ " voilà quelqu'un » sẽ " ça y est, on me l'a donné, c'est ouvert ", etc.

ul " ça roule ".

Dès cette époque, emploie des assemblages pour constater ou demander, voir plus loin p. 225.

20. Emploi de mot isolé : on le mène à mon bureau où je ne suis pas : dit d'abord $s y \ddot{o}$, un peu après pe : on comprend "c'est la place du monsieur (de grand-père), et il n'est pas là ".

$20+3$. Je note qu'il emploie encore $\tilde{e}$ modulé pour demander à manger. $20+4$. Emploie encore le souffle admiratif.

Dit toujours non de la tête.

Quelques emplois de $i, \ddot{w} i$ pour " oui " (qui n'ont pas persisté ensuite). Une fois papa? interrogatif " où est papa? " (première interrogation notée). 21. 3. Le même $\tilde{e}$ prolongé (non modulé) exprime le désir alors qu'il essaie d'ouvrir une porte.

Une émission nasale à bouche fermée, prolongée $(m)$, sert à réclamer un couteau. La même, énergique et moins prolongée, accompagne le "non » de la tête, qui persiste.

Il répète plusieurs fois $a-\ddot{w} i$; c'est sans doute issu de " oui ".

$212 / 3$. [Lettre de sa mère] s'il te plaît maman.

D'autre part a lexicalisé le rire : deux émissions voulues (dont quelquefois une inspirée) signifient " c'est drôle ».

Pour les clics suivis d'un mot parlé, voir p. 226.

$22+3$. Commence à employer le mot non.

$22+11$. Refus exprimé par $\tilde{e} \tilde{e}$.

$22+12$. Refus énergique exprimé par $a$ a $a$.

$22+17$. a a a exprime la demande.

$22+20$. (A dit to ato "je voudrais du gâteau " et n'en a pas obtenu) insiste en grognant $\ddot{a} \ddot{a} \ddot{a}$.

$22+23$. Demande exprimée par a a.

$22+29$. Refus exprimé par $\dot{e}$.

$23+15$. Généralement n'emploie pas encore le non parlé, mais le tourne ment de tête, et un grognement s'il s'irrite.

$23+24$. Emploie nõ ou nõ nõ nō " non "; ne dit toujours pas "oui »; énonce quelquefois ce qu'il consent à faire : "[tu veux monter?] mõte «monter».

$26+16$. Désirant quelque chose, grogne sur $\dot{e} \dot{e}$ en pleurant presque; ensuite fait une demande parlée sur un ton normal.

J.-L. 18. papo " on m'a mis mon chapeau ".

$p \tilde{e}$ " voilà grand-père (en portrait) 》.

$19+8 . a-i$ " c'est Jean-Louis" (dans la glace, en photo).

Question " tu vas te promener? ». Réponse nöne " oui, avec Renée ".

D'après témoignage de sa mère, raconte à une personne qu'une autre lui a donné quelque chose, par exemple mãmã a c'est maman qui m'a donné ca $x$. 
19 1/2. afé u le café qui était là tout à l'heure n'y est plus n.

"Où est Renée? ". Réponse : papo " elle va me mettre mon chapeau » (prévision).

ospe «c'est ouvert».

bube " je vais tomber » (sorte de prévision).

21 1/2. Demande : ato " je voudrais du gâteau "; mais le même jour, jouant avec son cousin : u u a $a$ a, énoncé sans colère, veut dire à peu près "donnemoi ce que tu as pris $)$.

Le même jour encore, emploi en terme isolé de ce qui est un verbe d'adulte $\checkmark \ddot{o}$ " je veux (bien) ", et de ce qui est une préposition d'adulte $d \ddot{a}$ " je mets la pelle dans le seau $n$.

Même jour : $p^{1} e * s^{\prime}$ il te plaît $», p \ddot{u}$ est un refus de continuer (" plus »). $21+22$. nö " non ".

$22+19$. [Tu veux mettre tes chaussons?] " non... pas ".

Conversation : osō " voilà mes chaussons ". - " Ils sont jaunes? " - Non (par geste?) už " rouges ", puis dam c'est-à-dire : « il y avait une dame quand on me les a donnés hier ${ }^{0}$.

ado " pardon $v$ (en passant devant quelqu'un).

Envoie un baiser pour dire au revoir.

none (au milieu d'autres propos) “ je viens d'entendre Renée qui a fait du bruit dans la cuisine $\%$.

$24+1$. nб " non $*$.

$24+22$. "Tu veux faire pipi?" nõ " non " Tout l'heure? we a oui ".

A 25 1/2. La première interrogation remarquée par moi : grand-mère? = « Grand-mère n'est donc pas là? Où est donc grand-mère? »

Ce petit choix d'exemples suffit à montrer tout ce qui peut être signifié par l'énonciation d'un élémecm ısolé. Répétons ici que des élémcoms de ce type subsistent un temps plus ou moins long après que les assemblages ont commencé à s'employer (1).

Il est important de noter que beaucoup d'énoncés ne peuvent être compris que par un entourage attentif. Pour d'autres, les parents renoncent à comprendre. Quelquefois ils pensent, peut-être pas toujours avec raison, que l'enfant a émis des syllabes sans signification. Si ce cas se vérifie, on peut penser soit à un jeu accompagné d'une malice (en général l'attitude de l'enfant dément cette dernière partie de l'interprétation), soit à une sorte de remplissage de sociabilité, l'enfant voulant absolument dire ou répondre quelque chose, alors qu'il ne dispose pas d'un énoncé correct. En général l'enfant ne paraît pas chagriné de l'incompréhension; le sentiment qu'il peut éprouver dépend de la vérification d'une des hypothèses émises ci-dessus. Pour le cas où il aurait

(1) A. Séchenaye, Essai sur la structure logique de la phrase, a dit, inaugurant, sauf erreur une manière de voir (p. 17) : - Dans l'acte de communication monorème normal, les circonstances données représentent le sujet par excellence de la phrase, et le mot, le signe linguistique usuel, est son prédicat.

Cette définition qui a été reprise par d'autres auteurs ne s'appliquerait à la rigueur qu'à certains des exemples. Elle introduit fàcheusement dans le langage enfantin des notions logiques de la grammaire, qui risquent de fausser l'observation, et donnent une interprétation abusive. 
en fait essayé d'émettre un énoncé correct (ou au moins intelligible) sans y parvenir, on pourrait se demander si l'incompréhension rencontrée le pousse à une certaine concentration sur lui-même ou au contraire l'incite à des progrès vers l'intelligibilité.

c) Éléments de communication en assemblages. - Si on tient compte des gestcoms, on constate que les assemblages sont employés assez tôt. Ils sont rares dans les débuts des exercices de communication. Ils augmentent ensuite en fréquence. C'est progressivement aussi qu'ils se compliquent de plus en plus.

Il se présente ici une question délicate : les assemblages, notamment les premiers assemblages à deux termes, peuvent comporter une interruption, une sorte de ponctuation (virgule ou deux points). Dans ce cas certains auteurs pensent qu'il s'agit d'un accolement sans fusion de deux actes linguistiques indépendants; il y aurait progression de ce stade à celui de l'assemblage proprement dit en un véritable assemblage (1). Cette vue ne s'applique pas facilement à tous les cas; la théorie exacte reste à faire, après des observations plus étendues; ci-dessous quelques interprétations sont discutées.

Pour l'appréciation générale tant pour les non-jonctions que pour les ordres des termes adoptés par les enfants et pour certaines significations, tenir compte du langage employé par les parents : "elle est partie, maman ", "partie, maman?", avec une virgule plus ou moins forte sont courants dans le langage "parent".

Le report à la division $b$ ) permet de voir à peu près à quels autres faits les premiers assemblages sont contemporains (2).

Fr. 19. "Maman est partie » est raconté en deux gestes : doigt tendu vers la porte, puis mains levées paumes en l'air.

19 1/2. toto puis lever de mains signifie : "fini de dormir ».

$20+4$. Premier assemblage entièrement parlé se trouvant dans mes notes : énonciation approximative əsiatati interprétée "le chien est parti ». (Noter qu'il n'y a pas de ponctuation.)

$20+11$. Une note semble dire que sur proposition " elle est partie, maman " a fait écho en ati ou etati mimm $\tilde{\tau}$ mais aussi par mãmãati.

Sur énonciation " tu vas tomber $n$, a répondu à peu près $i$ pö pa pape, interprété " je ne peux pas tomber ".

Déclare: papa li " papa est au lit ».

(1) C'est encore à Séchenaye, Structure logique, qu'il faut remonter, semble-t-il. A la page 19, il dit que la phrase à deux termes est la synthèse de deux monorèmes $j$ uxtaposés; page 37, il parle du dirème coordinatif (sans synthèse); une fois la synthèse opérée, les ensembles sont suivant les cas "principal-complément * ou a sujet-prédicat".

Marguerite Durand (voir p. 184) demande si le signe que la phrase à plus d'un terme est réellement constituée n'est pas l'établissement de la mélodie normale (montante puis descendante); des observations devront être faites dans cette direction; il faudra tenir compte en même temps de l'ordre des termes.

(2) Des exemples donnés dans Langages successifs. p. 122, trois sont repris ici en résumé. 
se s'o mẽ mãmã «c'est chaud la main de maman ».

ati li papa " papa a disparu dans le lit ».

$20+24$. A la question: “ qu'est-ce que tu as vu? ", répond par une énumération ponctuée de $\ddot{o}$ " euh " et avec répétitions : tita ö nana lolo ö nana lolo ö nana lolo ö bebe atür ö mãmã ö asõ " des trains, des canards sur l’eau (ter), des bébés en voiture, une maman avec ses garçons ".

21 + 12. adade toto "(je veux) regarder la photo ».

21 + 14. ave asi " on va laver Francis".

22 + 4. vwa asi " je vois (voir?) Francis (dans la glace) ».

$a$ pü ane "ça ne sonne plus ".

$22+17$. a fe papa? apum " qu'est-ce que papa a fait? Atchoum ".

o pase " je veux passer ».

L. 14 3/4. Assemblage composé de : äta avec un signe de tête, puis doigt tendu vers son père : il manque une traduction.

$15+3$. Autres assemblages : au réveil, lève un doigt en l'air en disant pape $(y)$; après avoir bu son biberon, montre la tétine en disant pa pa pa pa (avec des a chuchotés), ce qui semble imiter la tétée et ajoute papey; après qu'on lui a mis ses sandales, dit pape sur un ton interrogatif; plus tard dans la journée $p \varepsilon$ pe est identifié comme " chaussettes $n$. La traduction totale manque.

Plusieurs fois je note que des suites parlées sont incompréhensibles.

16. Après avoir uriné par terre : $Q 9$ pa po.

$171 / 2$. Pour refuser quelque chose $: n \tilde{o}, n \bar{o}, w \varepsilon \bar{a}(\varepsilon$ est une émission laryngale) " non, non - cri d'horreur imité de Fr. "

$183 / 4$. ple ple ko, assemblage net sans virgule notée, de sens douteux, " donne-moi des haricots" ou "s'il te plaît encore"; en revanche a pü " il n'y en a plus " est sans doute senti comme un seul élémcom.

$193 / 4$. lo.ta.pa (avec virgules), interprétation douteuse " de l'eau dans la timbale "?

popo a le le " voilà le pot " (on a par ailleurs alale " voilà »).

22 1/2. po ave (noté ainsi sans indication de virgule) " enlever le chapeau ». La note ajoute : première phrase? (il faut entendre : entièrement parlée et avec apparence de syntaxe). Ici, dans le cas où il y aurait eu virgule, on aurait pu admettre un accolement : "et mon chapeau? il faut l'enlever". Voir les cas nets ci-après.

23. Une conversation entière a été notée, à la suite de l'apparition d'une coccinelle sur la table : mãma op? probablement : « Maman, est-ce propre, acceptable? "; on lui demande "il y a une petite bête? "; en réponse, exercice sur "bête ", mot non acquis : $d e d, t e t, b e b, d e d$, puis : $\overrightarrow{u l}$ " elle a l'air de rouler ", at $\pi$ " va-t'en" ou "va-t-elle s'en aller? n, ayo "c'est un oiseau ", iye " elle va aller dans la cuiller ", pör "elle me fait peur ", be "elle va tomber", ape "il faut l'attraper $»$. (Noter que sauf la question du début il n'y a pas d'assemblages.)

On vient de raconter qu'elle a couru après sa mère au jardin; elle me raconte ensuite : $k \bar{u} r$ adè $m \tilde{\pi} m \tilde{a}$. (La notation n'implique pas de virgules.)

En regardant une image $s_{a-p o t i}$ (ici l'interruption est notée) a voila un chat; il est petit $n$.

Ensuite ge nu (pas de virgule notée), "je suis fatiguée; prends-moi sur tes genoux 
Pour lul-föf (une interruption a été notée alors que la phrase "adulte » n'en comporte pas: "la poule a pondu un ceuf ".

Récit fragmenté : $a n e-b \bar{o} b \tilde{o}-b e$ "grand-mère a apporté des bonbons dans le panier ".

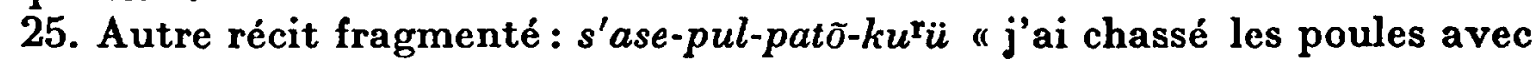
un bâton en courant ".

sé-ne " (il faut) moucher le nez ".

26. bsvär-ro-lo-abal « (je vais) boire du sirop et de l'eau dans la timbale . $^{\circ}$

$263 / 4$. božūr-sabl-påt-loẹ-tu, tu "bonjour, nous jouons au sable, faisons des pâtés, versons de l'eau; tout, tout ".

$272 / 3$. Récit d'un événement fictif, noté en orthographe française ainsi : " cassé, carreau - et puis - tombé "; le même jour, on l'entend dire, seule : " carreau tombé ct cassć ".

Les dernières notes sont du début de la période grammaticale. (Voir cidessous e.)

Mes notes ne me fournissent pas d'ensembles à termes nettement liés, qu'on trouve dans la période suivante.

Chr. 21. pipi popo "pipi dans le pot »?

21 1/2. Récit : tọm-tọm $\varphi(\partial)$ (sans séparation) "J'ai vu une dame. La dame m'a donné un chien. "

22 2/3. mama-po " maman a mis son chapeau [et elle est partie]".

23 1/2. lolo-po "Claudie est partie".

tam la toto "il y a eu une personne couchée là " (dans une baignoire vide désignée du doigt).

$l \bar{a}$-dodo "Francis se couche " (Pour la, voir p. 240).

$233 / 4$. alo puis alo tam, en montrant le second étage où elle avait vu une amie se tuber «la dame était dans l'eau».

papa-ana-dö "deux canards pour papa".

24 1/2. nana bebe-a (les deux premiers termes liés) “ voilà le nœud du bébé ").

28. iẽ lo la "il vient dans l'eau Francis".

28 1/4. ale mãmã "aller vers maman".

papa viẽ " voilà papa qui vient".

29. bebe lul mãmã " (voilà) un bébé que roule sa maman 》.

29 2/3. lolo vyê nene "l'eau me vient dans le nez".

Les documents donnent un mélange de ponctuation et non-ponctuation, où il est difficile de voir clair en raison du sens.

J. 17 1/2. En une période de vingt-quatre heures, je ne lui entends pas faire d'assemblages.

$19+6$. pati-tan " elle n'est pas là, Christiane ".

19 1/2. D'après le témoignage de Chr., les assemblages sont fréquents.

Une fois, à la maison : tü-lal, en montrant ses chaussures : "voiture-sale ๖ semble être un récit.

Observé par moi : kuku pe-ęt " te voilà, grand-père, tu as tes lunettes à la main ".

alę lep " (je veux) aller à l'herbe, là où il y a de l'herbe ».

[Se cogne le pied] pal o pye " (je me suis fait) mal au pied $n$. 
20. ul papa (sans intervalle) " roule, papa "!

ai ati (sans intervalle) "Marie est partie".

21 (-4). Témoignage de L. : depuis quelques jours dit, le doigt leve : $t \tilde{a} \tilde{o} \boldsymbol{o l}$ " entends-tu les garçons de l'école ».

ö pupẽ " je veux la poupée ".

21 (-3). yฮ mãma, en tendant les bras à sa tante "porte-moi à maman». nọn pupe mämã " je donne la poupée à maman".

pati työ « le monsieur est parti ».

apyum pe "grand-père a éternué $"$.

wa pe " je voudrais voir grand-père " (qui est dans la chambre d'à-côté). Deux clics médians suivis de tityan "Christiane mange".

ta $\bar{a}$ työ (en levant le doigt) "j'entends un homme »; puis pati työ (en écartant les bras) "c'est fini (je n'entends plus) l'homme».

apu ( $p$ )atõ " je mets le bâton debout 》.

tap pe " je tape sur la table, comme grand-père a fait ".

$21+5$. D'après $\mathrm{L}$. a dit à un petit garçon $e$ pul apr pati at ó \& ̀̀ Viroflay, vu les poules, les arbres; les chatons (de noisetier) [qu'on en avait rapportés] ne sont plus là ». [Récit détaillé].

Entendu par moi : to vö o " je voudrais le rond "; après l'avoir obtenu : ayi $\widetilde{o}$ " merci pour le rond " 'tu veux extrait de " qu'est-ce que tu veux ou de "tu veux venir" ou analogues est employé comme terme de désir; il remplace les grognements ou les cris précédents; se réduit au bout de quelque temps à $t \ddot{o}$, voir ci-dessous).

tutu up " j'ai mangé toute la soupe ».

nọ $p a-p a$ " je le donne à papa ».

L. a recueilli : non mãma papye papa "maman donne le papier à papa ${ }_{0}$ ef palo "enlève le pantalon".

22. Noté une fois $t \ddot{i} \varphi \ddot{o}$ au lieu de to $v \ddot{o}$.

$22+3$. ki $p q$ " il écrit grand-père ".

tutu pot-ni pọt " j'ai mangé toute la compote; la compote est finie ".

$22+7$. Témoignage de L. : ta fe pipi "j'ai fait pipi ", žefẽ : "j'ai faim ». 22 1/3. tapu ōm " moi Jérôme je veux monter sur le tabouret (exprimé par : debout).

$a \tilde{n}$ " (je veux) descendre ".

$221 / 2$. tavö mãze (ou mã̃e) la pọm " je veux manger de la pomme».

Emploi sporadique du ton interrogatif?

ole papa " où est papa?" (pas de ton interrogatif noté).

mõtr mãma " je le montrerai; je veux le montrer à maman " (anticipation). 22 2/3. to ato " je voudrais du gâteau " (comme on ne lui en donne pas, insiste en grognant $\ddot{a} \ddot{a} \ddot{a}$ ).

Ensuite ta est généralement employé; mais à $25+5$ on note encore à la fois $t a$ et $t \ddot{i} \varphi \ddot{o}$.

$223 / 4$. vivi pę " oui, grand-père ". (J'avais dû lui dire : oui? ")

23 1/4. D'une manière générale, s'il doit dire "oui ", emploie une énonciation entière : " tu as bien dormi? " fe toto " j'ai fait dodo "; s'il s'agit d'un ordre auquel il consent, il exécute sans rien dire.

[A 23. les notes portent : progrès brusque.]

J.-L. 19 2/3. Se tape dans les mains, puis dit me "fermer 》 et ferme une
boîte. 
Où est maman? Fait un geste de balayage, puis dit ati.

Dans les deux cas c'est soulignement ou doublement, non assemblage. 21 1/2. $u(v)$ fe " il a ouvert la fenêtre " (énoncé sans coupure). pa bü zožo, après avoir bu et voulant boire encore (sens?).

$212 / 3$. pope-ao "la poupée est là-haut ", "il y a une poupée là-haut » (énoncé disjoint).

(En montrant deux boutons) sa-dö « çà, deux ".

viv-vö « le livre... je le veux".

22 1/2. dam $i$ " la dame lit».

24. asi papa "assois-toi, papa " et aussi « [c'est] derrière papa qui est assis ".

pato $d \pi$ " les bateaux sont dans la poche".

[Une note dit : peu de phrases.]

$m \tilde{a} m \tilde{a}$ vsva mãmã "maman, je veux voir maman ".

bobo amyõ " le cheval en bois [baptisé camion] est cassé ".

sa if " voilà un livre".

24 1/3. Presque à la suite afe sö et vö afye $\mathrm{r}$ " je veux la cafetière ".

24 1/2. papa pati-te " papa part (est parti) prendre le train".

$242 / 3$. vö siñ " je veux le cygne».

ape mã̌ "grand-père mange ".

bör fadï " le beurre a fondu ».

25 2/3. me rör a poš papa " je mets le coureur dans la poche de papa ". asva dö bõbo sola " je veux avoir deux bonbons de chocolat".

26. Fréquemment répétition en queue du terme de tête. Ainsi, en entendant le bruit caractéristique : sa moto sa " ça c'est une moto "; en prenant ou montrant : sa patit fôy sa " ça c'est une petite feuille ".

טö $q$ " je veux du lait ».

mã̌ze tatin "j'ai mangé une tartine ".

Un assemblage particulier est no, maiza " non, Marie-Elisa ", dit à sa mère, avec une mine farceuse marquant sa conscience de faire une plaisanterie en l'appelant comme font les autres personnes.

Françoise Rouquès :

Exemple de précocité dans l'emploi des assemblages.

12. [Regardant une image de bébé au bain.] pla pla pf "bain-joli ».

13. lilila papa "il est là, papa ".

d) L'usage du vocabulaire. - Lorsque l'enfant ne pratique pas encore d'assemblages ou n'en pratique que peu, si certains termes ont un usage précis, correspondant a celui des adultes, d'autres ont une extension de sens beaucoup plus grande, qui se restreint à mesure que de nouveaux termes sont acquis. (Ceci se fait surtout parallèlement avec l'acquisition d'autres distinctions, en particulier des marques grammaticales, voir sous $e$.)

Fr. $18+9$ d'après mes notes comprend tout ce qu'on lui dit (ce qui sous-entend qu'on ne lui dit pas n'importe quoi); mais il n'emploie spontanément, d'après la somme du 21 novembre 1915 (voir ci-dessus p. 210) qu'une dizaine de termes; le même jour il est noté qu'il répète, apparemment en les comprenant, une vingtaine d'autres mots. 
L'enfant se sert au maximum des mots d'adultes ainsi acquis. Notamment ils apparaissent dans les gazouillis sans doute à l'état de monologue extérieur (à défaut de langage intérieur). Le même jour que cidessus, pour Fr., papa et koko (sans doute "gâteau ") étaient identifiés dans son gazouillis.

Il arrive que l'enfant signale quand il entend passer dans la conversation des adultes un des mots qu'il emploie ou au moins comprend; ainsi se trouvent isolées de manière inattendue certaines syllabes finales, plus faciles à percevoir.

A une date indéterminée entre 15 et 18, il est arrivé que J. entendant Natacha réagisse par " miaou ».

Voici un cas plus compliqué; du même sujet à $181 / 2$. Je disais sans précaution devant lui : "pour ça, il ne dépasse pas le stade du chien qui rapporte un caillou ": la réaction a été u, c'est-à-dire "chien " dans son langage : le déclenchement provenait sans doute de la finale de caillou; mais on ne peut pas affirmer qu'il n'avait pas identifié chien à l'intérieur de la phrase; les deux ont peut-être agi.

Par ailleurs les enfants, lorsqu'ils ont les possibilités d'articulation assez bien établies répètent' souvent en écho, à titre d'exercice, semblet-il, des mots ou des fins de mots:

$\mathrm{Je}$ disais, intentionnellement cette fois, des choses de "grande personne" devant J. à 22 mois; il répétait la dernière syllabe de chaque phrase; ainsi " je ferai une communication ". syô (pas très bien articulé). Devant J.-L. $221 / 2$ on parle sans s'occuper de lui du P.S. U. (nom de parti en abrégé); après un instant il s'amuse à répéter psü.

Les extensions de sens sont imprévisibles et ne sont compréhensibles que si l'enfant est suivi de près (1). Il faut tenir compte des homonymies qui existent dans le langage adulte et de celles qui se produisent en plus dans le langage enfantin à cause de la déficience du phonétisme et du raccourcissement des mots. Mais l'essentiel consiste dans l'utilisation extensive des mots disponibles, suivant des contiguïtés qui peuvent être des classements sommaires d'objets, en partie dues à des circonstances fortuites.

Pour Françoise Rouquès 13., vava est "chien, chat, fourrure "; l'origine est sans doute une imitation d'aboiement, car un mois après le mot alterne dans l'usage avec syẽ, pour les mêmes trois sens.

Les exemples qui suivent comportent des études sommaires de compréhension.

J. 20. "Monsieur " employé pour "grand-père " et rectifié, voir p. 221. 21. Dit af également pour "se coiffe " et "se lave "; il n'y a sans doute

(1) Des exemples sont étudiés dans Langages successifs, p. 115-120. Pour certaines des questions abordées ici et pour d'autres touchant aussi l'usage du vocabulaire, voir : Marguerite DurANo, - De quelques eliminations d'homonymes chez un enfant ", dans Journal de Psychologie, 1949, p. 53-63 (et B. S. L., t. 44, 1948, p. xx.1V-xxr.) 
pas de confusion pour lui, non plus que pour $\tilde{\sigma}$ " bouchon", "garçon" (voir p. 208).

A un stade d'acquisition beaucoup plus avancé (26) il a fourni un bon exemple de catégorisation, alors qu'il était en contact avec des choses nouvelles pour lui. Je ratisse et lui montre mon instrument : " qu'est-ce que c'est? " la faux; je dis : "non, un râteau "; il a l'air de réfléchir, et ne répète pas; un peu après, ayant une fourche en main, je refais l'expérience : "qu'est-ce que c'est? "; de nouveau : la faux; je dis : " non, la fourche "; cette fois, il essaie de répéter : d'abord furk puis furš. J'explique : "c'est pour emporter le foin "; après un petit temps, il dit " coin " (mot qu'il connaît : pour " balai " voir p. 245). Autre observation : il a vu des lézards, mais non des cornichons; j'en cueille deux que je mets sur la table, il dit spontanément "les deux lézards "; je lui dis : "cornichon ", qu'il répète correctement; quelques minutes après, il recommence à employer "lézard ".

A la même date j'ai fait des petites expériences, pour voir comment il réagirait à des mots non connus, et présentés sans explication, et qu'il est matériellement capable de répéter correctement ou à peu près. Je lui dis : " c'est extrêmement chaud ", il répond "c'est très chaud ". Je dis : " tu as vu qu'il faisait du soleil et tu as conclu que c'était l'été »; il répond "il fait soleil ». Les deux fois, il s'en est tiré en comprenant une partie de l'ensemble. Je pousse l'expérience en lui disant : " tu es allé au jardin; as-tu fait de la botanique "? Il répète bolanik d'un ton affirmatif et c'est tout. Il n'en est pas encore au stade de la question "qu'est-ce que c'est "? Mais il est possible que le mot ait été retenu et soit obscurément reconnu plus tard. (Sur certains faits de mémoire, voir p. 212).

Trois jours après, une expérience, involontaire celle-là au moins dans le début de son exécution, m'a montré des manques de compréhension inattendus, portant à la fois sur des attitudes claires et des mots simples. Voici toute la scène. Cueillant des navets alors qu'il est à côté de moi, je lui en donne pour les porter à sa mère et je lui dis : " qu'est-ce que c'est? ", réponse poireau; " non, navet "; il ne répète pas, mais dit porter à maman (à l'opération suivante, c'est devenu : je porte à maman); puis je lui dis : "il y en a encore, ils sont là ", en montrant du doigt; il répète là, ne regarde pas au bon endroit et ne voit rien; je dis : " regarde, devant toi "; il répète " devant toi ", sans résultat; alors : "avance un peu "; pas de résultat; "viens ici ": il comprend et il avance; "arrête-toi ": il comprend et s'arrête; "les navets sont sous tes pieds "; il ne regarde pas, bien que gardant un air de bonne volonté, d'intérêt et même de réflexion; je renonce et il s'en va; mais au bout de peu d'instants il revient, prend les navets et les emporte sans rien me dire. La conclusion est qu'il vaut mieux ne pas écrire d'un petit enfant qu'il comprend tout ce qu'on lui dit (voir ci-dessus, p. 227). D'autre part, il y a des chances pour que dans un cas comme celui-ci une partie des termes non compris qui affolent plus ou moins l'enfant, de sorte qu'il ne paraît pas réagir, soient dans une certaine mesure enregistrés, de manière à servir par la suite.

L'incompréhension peut être soulignée par une confusion : ainsi à L. $171 / 2$ on disait (non pour la première fois) "fais des grâces "; elle a réagi en faiš́án des grimaces.

L'accroissement du vocabulaire devrait être mesufé Ánssì sonvent que 
possible chez chaque enfant, en séparant les mots employés (spontanément) et les mots compris et si possible en notant les disparitions éventuelles. Malheureusement on en trouve rarement le loisir.

A partir du moment où l'enfant a réellement démarré pour parler, le nombre des mots qu'il emploie est plus élevé qu'on ne croirait en commengant la collecte.

Une somme tentée pour J. 22 (mots employés) a donné un total de 320; une autre pour J.-L. 26 (également mots employés) a donné 495 . (Collecte par la grand-mère, révision et classement par les parents respectifs; pour l'utilisation de certains aspects de la composition de ces vocabulaires par le grand-père, voir la division suivante) (1).

e) Les débuts du passage au langage adulte. - Nous avons envisagé jusqu'à maintenant un état linguistique très éloigné de celui du langage de l'adulte; il s'agit maintenant de considérer le passage de l'état proprement enfantin à l'état adulte.

Ce passage se fait progressivement, et tant qu'il n'est pas entièrement réalisé on peut dire qu'on se trouve encore en face d'un aspect du langage enfantin.

Tout langage complet est différencié et présente une structure, qu'on décrit généralement en gros sous le nom de grammaire. Désormais nous devons parler de mots et de phrases, d'espèces de mots et de rôles des mots dans la phrase.

Cette différenciation du matériel linguistique correspond à des opérations mentales qui ne se réalisent qu'à partir d'une certaine étape de la croissance, en répondant à des besoins croissants en complication de l'activité individuelle et en groupe, et des communications a ce sujet.

Les distinctions qui se reflètent dans les grammaires et les vocabulaires sont variées suivant les langues, émanations des diverses sociétés qui se réalisent en nombre indéfini au cours de l'évolution humaine : elles sont le résultat de circonstances extrêmement complexes et ne correspondent ni à des facultés innées immobiles ni à des concepts universellement valables (2).

Tout acte linguistique parlé se compose de phonèmes dont la liste et le système plus ou moins symétriques (et à symétries de sortes différentes suivant les systèmes) n'apparaît que lorsqu'une langue est décrite méthodiquement. On parle sans prendre conscience de l'ensemble

(1) W. F. Leopold a noté en 24 mois 8 mots $\alpha$ non-standards $», 260$ mots anglais et 160 mots allemands avec leurs dates d'acquisition; défalcation faite des disparitions (surtout par remplacements) le total restant à la fin de la deuxième annéc était 43 mots allemands, 46 à la fois allemands et anglais, 152 anglais, soit 241 (195 si on défalque ce qui est seulement allemand). L'observation faite par A. Grégoire au cours des mois 19-24 a donné 132 mots pour un garçon, 113 pour l'autre; au cours des mois 33-38 le total a été pour l'ainé de 536 ( +758 compris non employés spontanément), pour le cadet de 447 .

(2) $\Lambda$ cet égard, à la date où ceci est écrit, il faut mettre spécialement en garde tous les travailleurs en matière de langage contre les livres de Georges Galichet, Essai de psychologie linguistique, et surtout Physiologie du français. 
phonologique de la langue, de même qu'on n'a pas la conscience détaillée des mouvements musculaires qui servent à produire ces phonèmes. De la même manière c'est inconsciemment que la plupart des hommes pratiquent et transmettent à leurs enfants la décomposition de la phrase en sortes de mots différentes et différemment agencées les unes par rapport aux autres et la décomposition d'une partie des mots euxmêmes en parties jouant des rôles différents. On distingue essentiellement celles qui désignent des sens, en particulier des objets et des " procès ", c'est-à-dire des actes ou des changements d'états, et celles qui distinguent des notions appartenant à la fois à de nombreux mots de sens variés, ainsi la pluralité, l'appartenance à une personne, la dépendance par rapport à un objet ou à une action.

Comme tout se tient dans la croissance, il y a interdépendance des divers progrès du langage en cours d'acquisition.

Lorsque l'enfant possède tout ou presque tout le stock des phonèmes, qu'il peut les tenir distincts et à leurs places dans des suites assez longues (syllabes complexes, mots de plusieurs syllabes) et qu'il peut renouveler plusieurs fois de suite cette opération pour constituer une véritable phrase, et pas avant, l'enfant devient capable aussi de commencer à assimiler par imitation les procédés adultes.

Les psychologues ont à examiner ce qui intervient alors en fait d'activités d'attention, de discernement, de mémoire, de raisonnement par association, etc., de manière à ce que des détails précédemment négligés au cours de l'audition soient perçus, et ensuite utilisés activement.

L'analyse du comportement de l'entourage doit intervenir en même temps que celle du comportement de l'enfant. Avec un enfant qui comprend plus, répète mieux, exprime plus de choses spontanément, les parents s'abstiennent de plus en plus de certaines simplifications qui sont naturelles, même chez ceux qui ont la volonté d'en user le moins possible. Disons d'une manière générale que les rapports et la conversation deviennent plus nuancés.

En même temps s'augmentent les capacités de l'enfant pour percevoir des détails au vol dans la conversation des grandes personnes et des enfants plus grands.

Au cours de la constitution des langages développés des enfants, $\mathbf{1}$ intervient normalement certaines contagions dues à des contacts plus ou moins fugitifs qui échappent à l'attention de l'entourage (1).

Comme les autres étapes, le passage à un langage adulte encore imparfait commence à se faire à des âges différents suivant les individus et il ne se fait pas d'un coup, mais d'abord par des apparitions sporadiques de certains phénomènes : au total, d'après les observations faites jusqu'à présent sur le français, l'anglais, l'allemand, il semble qu'on

(1) Voir certaines indications a ce sujet dans $A$ propos de la $3^{\circ}$ personno du féminin pluriel en frangais. 
puisse le situer au plus tôt un peu avant la fin de la deuxième année plus généralement dans la première partie de la troisième.

D'après ma documentation, j'ai été amené à réunir ici des phénomènes qui ne sont pas homogènes au point de vuc des descriptions habituelles soit linguistiques soit psychologiques; ils impliquent tous des analyses, et des activités correspondantes, en relation avec un même stade de croissance. Encore ici donc, il n'est pas procédé pour les enfants comme pour des adultes. Je ne sépare pas les phénomènes dans des listes d'observation distinctes. Mais les listes d'exemples ne seraient pas compréhensibles sans un tableau préalable, au moins approximatif des différentes catégories qui y sont entremêlées.

Présence et emplacement. - Les gestes démonstratifs sont précoces; certains adverbes peuvent les remplacer assez tôt. Certaines prépositions répondent au rangement dans l'espace.

Absence. - Les gestes indiquant l'absence, le départ, sont précoces. L'emploi de la négation accolée à un élément principal est à ranger ici.

Le passé exprimé par une flexion verbale ou par un adverbe peut se rattacher au même ordre d'idées.

Quantité. - Les notions de quantité et de répétition ne sont sans doute pas à séparer; elles peuvent apparaître au stade des élémcoms isolés dans certaines expressions d'admiration et dans des parlcoms issus d'adverbes, surtout "encore ". Des emplois de "deux", éventuellement d'autres noms de nombre mal compris, et d'articles pluriels, sont à ranger ici; sans doute aussi les adjectifs de dimension.

Couleurs. - Sans doute à distinguer des autres qualités et apparaissant de manières inégales dans l'expression comme dans la perception.

Propriétés diverses. - Il faudrait sans doute distinguer diverses catégories : chaud, froid; douceur, rudesse au toucher; gentillesse, méchanceté. La saleté est spécialement importante. Ce qui constitue pour l'adulte des qualifications apparâit déjà dans les premiers stades, comme élémcoms de différentes espèces. Il n'y a qualification véritable qu'avec l'apparition d'adjectifs épithètes. Ceux-ci sont insérés dans les listes ci-dessous comme se référant au même stade que les articles.

Individuation, identification. - Il s'agit ici de notions assez difficiles à définir et à bien distinguer. La notion de "autre " est connexe à celle de la pluralité.

L'identification des individus, y compris le sujet lui-même, est préliminaire à l'usage des distinctions des personnes (moi, toi, lui).

Les articles du français entrent dans cette division, en même temps que dans celle de la quantité : on peut souvent considérer l'article singulier comme un "singulatif " par rapport au pluriel ou au collectif.

Personnes (les unes par rapport aux autres). - Notions exprimées par les pronoms personnels indépendants, et par les pronoms adjoints au verbe, et par certaines terminaisons verbales (1).

1) Dans Rostand, p. 117-118, un utile tableau résumb́ de l'apparition des pronoms personnela, 
A l'occasion, il peut y avoir distinction des êtres animés et d'objets inanimés. Dans la grammaire française ce n'est pas normal, malgré l'usage de quelques pronoms impersonnels (" que " " ca ").

Rapports de situation, appartenance, destination, comparaison, etc. Ce sont les rapports exprimés en français par les prépositions et certaines conjonctions de coordination et par des conjonctions de subordination quand il s'agit de situations et d'actions complexes.

Époques, achèvements, inachèrements. - Se rattachant plus ou moins à la présence et à l'absence. En français ce qu'on appelle temps de verbes marquant des temps absolus et relatifs et des aspects de durée, de résultat, et le contraire (fait momentané, inachèvement).

Volontés, souhaits. - Exprimés de bonne heure dans le langage enfantin, surtout par des gémcoms et des gestcoms puis par parlcoms seuls et en assemblages. Dans le verbe, impératif, conditionnel.

Curiosité, besoin de nommer. - Sur l'interrogation, voir p. 219, 221, 222, 224, 226, 244.

Dans les langues, qui se forment inconsciemment au cours de l'évolution, il n'y a pas de structure entièrement régulière. Toutes sont difficiles à manier. D'autre part toutes sont en évolution constante encore que lente. L'enfant se trouve donc normalement d'une part en présence d'un système qu'il perçoit inconsciemment de manière plus ou moins complète, d'autre part en présence de certaines irrégularités : celles-ci sont en partie des valeurs multiples dans une seule forme (l'homonymie morphologique, dont on parle généralement peu), en partie les moyens matériels différents d'exprimer une même notion dans des mots de même catégorie (en particulier les exceptions).

Lorsque l'enfant nivelle les exceptions, au moyen de l'analogie ( les chevals ", "prendu " comme "rendu ") on a une preuve que le sentiment du système ou d'une partie du système est établi.

On considère que la période de petite enfance est terminée quand ces sortes de formations analogiques régularisatrices sont toutes ou presque toutes éliminées, l'enfant adoptant les irrégularités inculquées par les adultes.

Il faut savoir d'autre part que certaines des régularisations viennent à être tolérées par les adultes à certaines époques de l'histoire des langues; elles persistent alors chez les enfants lorsqu'ils sont grands, et ils les transmettent à leurs descendants. (Exemple simple : la différence des voyelles de : j'aime, nous amons de l'ancien français a été éliminée au profit de la conjugaison uniforme : j'aime, nous aimons.)

Dans les' tableaux résumés qui suivent, seuls figurent des exemples choisis; les faits nouveaux sont mis en valeur par la mise en italiques dans la "traduction". Le mélange de l'ancien, qui subsiste par ailleurs 
plus ou moins, et du nouveau est faussé dans cette présentation, qui ne fait pas apparaître le chevauchement des stades. Il faudrait des enregistrements complets de suites de propos. La notation phonétique montre quel est à cette étape le degré de correction de la prononciation.

Fr. $20+11$. ati tu tu tu "petit toutou 》, ati tato "petit gâteau », ako pati - encore pied, l'autre pied".

21. akẫr pade "l'autre pied".

de ta " des trains", de tetatar " des pommes de terre".

$22+17$. e so "le seau " (un seau?) a pe "la pelle ", eme la pop "fermer la porte ", a a $b a$ " je veux la balle ".

Remarquer les mots d'une syllabe : il semble que l'article apparaisse plus tôt avec de tels mots, par tendance à constituer des mots plus longs.

a fe papa? "qu'a fait papa?".

23 - 3. ün det " une lettre ", a po " un (le?) pot", a tito " un (le) bouton 》 (ici, il ne s'agit pas d'un monosyllabe).

23. a dün "la lune ".

$23+3$. etetet "des (les?) chaussettes ".

$23+6$. pipi a li "pipi au (dans?) lit".

$a k o^{r} f e$ " il faut encore le faire".

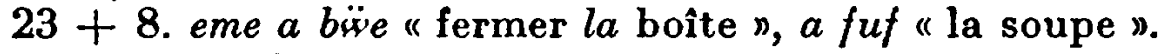

$23+9$. a fal " un cheval ».

$23+12$. a pus " je pousse ", a fe "qu'est-ce qui fait "(?).

$23+17$. a bül "çà brûle ».

$23+18$. pasi " merci ».

$23+20$. a lili mãma "aller dans le lit de maman 》.

a pa apa " je ne peux pas l'attraper " a pa tŭfö " je ne veux pas de chou fleur " tyęn " de la crème ".

24 - 5. a ba da la $l_{\ell}$ " je veux boire $d u$ lait".

[Forme remarquable, avec $d u$ décomposé; ce qui suppose à la fois le sentiment de l'article et de la préposition; il est possible que ce soit emprunté à un autre enfant.]

a ti daso " un petit garçon".

$24-3$. tï süs " $d u$ sucre ".

On note un emploi correct de si en réponse à " non ".

Une fois tosi " aussi ".

ellu "c'est lourd "[remarquer l'emploi d'adjectif-attribut].

et $p a$ " $d u$ pain ".

abål do asis "la timbale de Francis" (noter l'emploi du nom pour se désigner expressément).

$24-2$. alla la atür " elle est là la voiture ".

elela (e le la) " il est là 》.

a apel "il s'appelle ".

$26+5$. [Notes de sa mère, avec figurations en orthographe française, que je retranscris en écriture phonétique.]

mãma a mi dï bör dasü " maman a mis du beurre dessus 》.

e pi alors "et puis alors".

asi ta tütöy " assis dans le fauteuil " (" dans 》 est douteux).

a tutalör "à tout à l'heure ". 
mãma ton lo le a svatis " maman donne le lait à Francis».

pèta " maintenant".

sisi för " celle-ci de fleur " " cette fleur-ci? 》.

põte sü " monter dessus».

(Absences de prépositions : " pas fait pipi culotte du tout du tout 》; " maman chercher lettre papa "; " prendre sucre cuiller ».)

nene tapi ta mämã "donner le tapis à maman ".

pet te sis dadâ?? "mettre des cerises dedans?" ".

Nombres récités dans l'ordre : 1, 2, 3, 5, 7, 14, 10, 9, 4.

fafuf nspar "pantoufles noires".

koñe la mẽ " cogné la main ".

akor a pö do stape "encore un peu d'eau s'il te plaît ».

dame " jamais".

pęt pel ta so "mettre la pelle dans le seau".

pas vilè-sac " pas vilain-sage".

var męmęn yẽ " (je vais) aller voir (si) Maimaine vient".

[Première phrase notée à deux propositions.]

$f(x) \tilde{s i s}$ a mi tu săl sasur "Francis a mis $d u$ sable (dans ses) chaussures ». pö do pé avęt " un peu de pain avec ".

paka "parce que".

a kar to petye papa? " papa (est-il) $\grave{a}$ (la) gare de Poitiers "?

$e k a$ sisi " il est grand celui-ci ".

27. Sa mère note, sans exemples, qu'il emploie les articles le, la, des.

$28+7$. Notation par moi en orthographe française.

[Apparition de certains pronoms.]

Veux bien, toi tu fasses un g(r)os pâté, toi; un très $g(r) o s ;$ est un très g(r)os, ce(lui) là.

Est la fumée ça, il se sauve [issōo].

Où donc qu'il est bête? = où est la bête?

$28+8$. voudrais une cuiller. [Note : je n'existe pas.]

Moi ai couri (pour couru).

[Note : il est courant; mais le pronom sujet manque souvent.]

$28+9$. Pas pour toi, est pou(r) moi.

[Note : emploi courant de l'impératif, singulier, en concurrence avec l'in.

finitif]; exemple donné : $v a-t$ 'en.

pardon!

C'est un cheval qui fait ça.

na $d \ddot{o}, t w \bar{a}$ " il y en a deux, trois ".

Monter dessus [sü].

Moi je [za] vois. [Note : apparition de " je ».]

Mais ensuite : moi ai monté, moi ai dit.

ta se sa "qu'est-ce que c'est que ça ".

C'est toi.

Où $i l$ est maman? il a mangé.

$28+10$. "Moi est pas vilain; papa pas fâché parce que [pakə] moi pas vilain. "

Mais ensuite "moi ai tombé a rue moi ai couri ["parce que" manque].

Emploi d'impératif pluriel : "venez, attendez "; quelquefois "vous " au lieu de " toi "; phrase "vous a des bas" = tu as des bas. 
"I(l) s(e)rait monté." Note [pour "il sera monté »?].

$29+3$. [Noté par sa mère.] Tu descendras, tu? Tu as mesoin [= besoin]?

$30+4$. [Noté par sa mère.] Vous, tu discends?

["Pour vous" et " pour toi " indifféremment.]

On va faire loui marcher.

Moi, le ferai plus.

Demain moi irai à la Mérigote; moi étais hier. (Emploi de futur et imparfait.)

$\mathrm{I}(\mathrm{l})$ veut pas (ve)nir.

$X$. et $Y$. viendront.

I(ls) sont (ve)nus tous (I)es deux.

Voudrais du sucre [süt] s'il te plaît [sitope]. Maman.

Moi vois; toi aussi tu vois?

[Possessifs, avec confusions.] Ca c'est mon livre (= c'est ton livre); moi veux ma mienne; mais non, c'est ta mienne.

Moi ai mangé; moi me coucherai dans mon petit lit et puis mettrai mes pieds sur ma boule.

Regarde la voiture qui a deux roues [rut $]$.

Ce papier il est à moi.

Ca c'est un (pe)tit oiseau qui s'appelle corbeau.

Tu mettras moi dans la (v)oiture quand on sera sur la route de...

$30+18$. $\mathrm{M}^{\mathrm{me}} \mathrm{D}$. raccommode [tomod] son chapeau de Marie.

Si tu le fais encore une fois, te mettrai à la cave.

" J'ai dit à Michel "; mais ensuite " moi ai donné à manger au cheval ", et ensuite " on va donner du pain chocolat à les petits enfants". "Le béret de moi "; "il est $\dot{a}$ moi ».

$31+4$. sri et za swi " je suis ».

"Je " manque souvent : "ai bien dormi ", " ai sommeil ".

ma ka " parce que ".

Il s'en va $=$ elle s'en va.

"C'est la moutarde qui a me fait mal " [qui m'a fait].

31. "I(l) a me pas dit oui " [il ne m'a pas dit...].

A cette date : emploie un langage de mots inventés; des phrases fictives souvent terminées par un mot français; les sons sont peu variés.

$32-3$. Mon tien est pareil que celui de X.

$32+16$. Tu vas voir, je vais te montrer.

La boîte est pas ouvrie.

$35-3$. Somme : toutes les articulations acquises, cependant les chuintantes ne sont pas toujours correctes.

Présente un chantonnement poitevin (élevé à Poitiers, par sa mère parisienne).

Bon usage des formes verbales, des pronoms relatifs, etc. Cependant je quelquefois absent, ou remplacé par moi, moi/je.

37 - 5. Progrès notables. Quelquefois encore zézaiement.

Petites fautes : maska pour "parce que".

$40+6$. La note dit : langage acquis - avec accent poitevin.

Très peu de particularités notées. 
Choix de notes sur la sixième année :

Faisez pas de bruit - désembarrassé - c'est moi qui le fera finite (= finie) - les deux sont entre toi (= tu es entre les deux) sourier (= sourire, verbe) - il disparaitait - j'ai peinturé - il boivait - elle sera nétonnée - tout de suite et sans manqtion - je suis mal dans mon aise - je mours de chaud - [abréviations originales] pap = papa, caf $=$ café - il contenira - ça valerait - pour ma première fois de ma vie que je fais des additions.

Le dossier comporte un dessin avec bonshommes fait à 5 ans et $1.1 / 2$, et une espèce de chanson parlée composée à 6 ans et 9 jours.

L. $19+16 k o^{\mathrm{r}}$ " encore ".

$211 / 2$ by $\widetilde{e}$ " je voudrais être bien (sur le pot)".

22. potit "petite" appliquée à une épingle plus petite que celle dont elle a l'habitude.

23 - 5. lal "(je suis) mal".

23. $s^{\prime}$ os "Laurence ".

$241 / 2$. Fr. a entrepris de lui apprendre " moi, toi ». Elle me dit $l i$ «aller dans mon lit "; je lui demande : Christiane? Elle répond as "Laurence "; je lui dis " toi? "; elle répond tya " toi », puis se reprend : mwa, mwa " moi ».

Le lendemain emploie a m\&a "à moi », de même le surlendemain, ensuite a tan " à Christiane".

patu " partout".

24 2/3. tya " trois " tratr, tatr " quatre " (emploi pas indiqué).

fat $\tilde{\alpha}$, vat $\tilde{u}$ " va-t'en" et tale " t'en aller!".

otr " autre".

25. ofrō " au front ".

es le " laisse-les".

pafiy " ma fille".

mwa " moi " (je vcux lc faire).

tu s'a " tout ça".

$y \bar{o} n$ " jaune ".

$25+8 . i$ " oui 》.

akö "la queue 》.

dodo, a pa " je n'ai pas voulu dormir ".

$25+18$. babyẽ "pas bien".

26 - 3. Se désigne par $\tilde{a} s$, les autres personnes par leur nom; pas de pronoms acquis (voir ci-dessus).

$p u p a$ " je ne peux pas", se pa " je ne sais pas".

A ce moment on note une avance dans son langage en général (substitution de nouvelles formes pour des mots antérieurement acquis, ainsi panye au lieu de ane "panier").

apuš « la bouche ".

aföf " un ouf ".

$26+6$. atode " attendez " (pour " attends ").

rẽ " rien".

pavre "pas vrai ".

$26+13$. sal " sale ${ }^{\prime}$. 
$26+16$. akyor " encore".

$26+17$. A depuis quelque temps déjà le sentiment de la préposition " à »:

à papa, aux poules...

sęla " celle-là ".

si, en réplique à non.

$26+24$. lo "de l'eau 》.

27. asck "avec".

gad " regarde".

$27+6$. Emploi d'un article $a$ " un " ou " la "?

$27+13$ önöf " un œuf ». Semble n'employer ces articles qu'avec les mots courts.

$27+14$. ku twa couche-toi.

ofè enfin.

$27+19$. (a parlé d'un chien; on lui demande s'il est noir) a blä " un blanc ».

$27+20$. Voir p. 225; emploi de et (puis).

pa ma (ou $m u$ ) sa "pas comme ça."

Jeux sur " encore " akkikọkor, akọkiyọ, akiyọ.

$a$ ou ô gasĩ " un garçon".

$27+21$. pakisqt (t prononcé? ou erreur de notation?) " je ne sais pas qui c'est ").

$28-2 . i$ plö "il pleut " $i$ plöve " il pleuvait, il a plu ». (Distinction du passé).

$e$ est employé pour " les ».

28. $\ddot{u}$ ku.̌ " une couche ".

Prend un collier et dit mętr " mettre " puis za me " je (le) mets".

$28+5$. ta vï " tu as vu? " (après avoir fait une petite acrobatie).

tii v\$a " tu vois? " (en montrant un livre d'images).

$28+7$. e $m \tilde{a} \tilde{z}$ " (je) les mange".

$28+9$. ze trupe " j'ai trouvé 》.

Montre sur moi : "deux cols - un blanc, un noir - pas trois".

$28+21$. [Notes de la mère.] Laurence crie toujours, elle (Christiane) pas toujours.

$29+8$. Titane veut pas le rendre $\grave{a}$ moi.

$29+10$. bablement (probablement). Crois-tu?

[Note de moi : $\tilde{o}$ pis $p u^{\mathrm{r}}$ mswa " un pain d'épices pour moi ».

$29+12$. Successivement "mon nauto, ma l'auto, ma nauto".

$j e$ ferme; $s a$ tétine.

$29+13$. tombera $=$ " je vais tomber " passe (impératif).

$29+14$. dans le jardin, au sable.

$29+17$. sommeil, $t o i=t u$ as sommeil. "C'est mouillé dans la chambre

à maman ).

aller au jardin, maman, dis.

$30-6$. le l'ogre; le (= je) l'aime.

$k \ell ̨ k s a$ " qu'est-ce que c'est que çà ".

$30+1$. [Notes de moi.] : Grande période d'essai.

Conversation du matin : " Dire bonjour à maman? Bonjour maman. Entend pas. Bouge pas. A côté papa. Je veux pas à côté papa. " "Sales, mes chaussures, pas les mettre, des pantoufles [j'ai des pantoufles à la place].

$30+6$. Un peu plus, tombé [je serais tombée] dans le bain. 
$30+10$. Invitée à dire : " tu nous casses les oreilles $》$ dit " tu casses les oreilles à maman et à moi ».

je veux les siennes à toi (= les tiennes).

pourquoi jettes-tu ça (auparavant : tu jettes).

je vais au jardin, n'est-ce pas?

[Reprise de mes notes].

$31-2$. quelle couleur? (de quelle couleur est-ce?). Intéressant par l'interrogation, et pour le sentiment de la couleur.

$31+5$. c'est bon papa qui a l'acheté.

$32-3$. du velours bleu.

$33+16$. donne les ongles qui sont dans ta poche (= la lime à ongles qui est...).

$34-10$. veux persiste au lieu de " je veux $n$; je est généralement employé ailleurs.

$35-8$. wim = oui, maman (par jeu).

usrii " ouvert".

$35-2$. Note : en général les temps et modes sont corrects, mais : " on rira avec Fl. et puis on a couru " (= on a ri).

$35+12$. " tu m'as fait mal à le bras ", corrigé spontanément en " au bras ».

$35+17$. j'ai prendu.

$35+20$. quand je m'en vais être grande (= " je serai ").

$36+16$. c'est moi m'en vais le souffler (= " qui vais ").

$37+15$. dès quand je suis réveillée [puis correction] dès que je suis réveillée, j'ai demandé à faire pipi.

38 - 7. prendu (= pris), ouvri (= ouvert).

39. paka, pasko "parce que ".

$39+1$. un nègre, (pluriel) des ègres.

$39+16$. je vais te donner mon sien.

40 - 8. je marchera-à les deux (= aux deux).

$41+13$. vous irons (= vous irez).

$41+20$. je me suis tait (= tue).

ils mangent tout qu'on mange nous (= tout ce que nous mangeons).

42. si j'en aurais.

elle est beaute, corrigé en beau puis en belle.

43 (environ). tiendre (= tenir).

$44-3$. j'ai rêvé qu'on jouyait - ensuite dit : on a joué.

$48+17$. "Quand on est un monsieur et qu'on travaille on est un ouvrier; quand on est une dame, on est une ousrielle; quand on travaille au jardin, on est une jardinelle."

4 ans 2 mois. Dit souvent demain pour hier.

4 ans 2 mois $1 / 2$. elle tienait pas (= ne tenait pas).

4 ans 3 mois. je suis une faimseuse (= quelqu'un qui a faim).

4 ans 4 mois. quelle chaudeur (= chaleur).

4 ans 5 mois. ça servrait (= servirait); ça finisserait (= finirait).

4 ans 6 mois. ôte-toi que je boye (= boive) mon thé.

5 ans 3 mois. Elle est timide contre (= à l'égard de) X.

ils sont dures les cerises (1).

5 ans 5 mois. solfégéter "faire du solfège ».

(1) Pour la persistance de cette non-distinction de genre jusque dans la $10^{e}$ année, voir l'article dans $B . S . L ., 1927$. 
6 ans 4 mois. pour qu'on puive aller.

6 ans 6 mois. j'en voudra (futur; répété avec insistance).

6 ans 7 mois. Constamment à les pour aux; commence à se corriger ellemême à 6 ans 8 mois.

6 ans 10 mois. j'ai à tes souhaité (= éternuć, à cause de la formule " à tes souhaits $)$.

7 ans. voudrais des pommes de terre. (Certaines suppressions de pronom semblent provenir de la contagion de sa sœur plus jeune d'un an.)

7 ans 1 mois. un animau (1).

7 ans 10 mois. il vieillissera.

De 8 à 13 ans. Les observations sónt en nombre infime (21 dont 7 pour ils-elles).

Chr. $22-6$. $d \ddot{o}$ en montrant deux ânes, deux oreilles.

$22+2$. des pincettes sont d'abord appelées "canne " puis deux cannes. $22+10$. atō encore.

23. On lui énonce les premiers chiffres; répond : $o, t \ddot{~(2) ~ p u i s ~ n o ̃ ~ " ~ n o n ~ ", ~}$ tat (4) puis no, wit (8) (9 et 10 ignorés) om (11).

27. Se désigne clle-même par " oui ", sa sœur et son frère par la " là ".

$27+6$. Compte " un, deux, huit "; on lui dit : tu savais compter 1, 2, 3, 4, répond : " non, un, deux, quatre".

Vers 28. Note : se met décidément à parler; répète ce qu'on lui propose.

28 - 7. Répète quand on compte, jusqu'à 20.

$28+2$. pul pitit " petite pelle " (peut-être "la pelle est petite "?).

$28+5$. Commence à dire oli, lolol "Laurence " ati atti "Francis".

$28+10$. A peu près s'it "Francis" et pour la première fois tan "Christiane ".

28 + 11. Adverbe, négation : ñ̄ byõ dot " je n'ai pas bien sauté ".

$28+17$. talö " tout à l'heure " (passé et futur).

29 - 6. be pum epi plö " je suis tombée et puis j'ai pleuré ».

bobõ pus plii böbō mõ " j'ai mon bonbon dans la bouche, je ne l'ai plus dans la main ".

$29+2$. ma " moi" (phrase pas indiquée).

29 + 5. avęl tutet "la pantoufle s'est enlevée avec la chaussette".

30 - 7. pa " toi " (signifie aussi "trois "; "fois ", " froid "); il n'est pas question de "pas", qu'on trouve employé ensuite.

$30+11$. pö pa yuyi po " je ne peux pas ouvrir la porte ".

32 + 7. Emploie couramment paka " parce que "; ex. : nõ ale dã lo paka monik plö (ou plör?) " ne pas aller dans l'eau parce que Monique [la poupée] pleure ». Premier exemple d'article ("l'eau »).

$32+10$. Une partic des mots avec article, emplois de prépositions, adverbes, pronom personnel :

dĭ la bĩp a pa "dans la chambre à toi ", dal da bì " salle de bains ", dã $l$ boïz " dans le moïse ", di lo dad" " dans le jardin", dã la bamiz " dans la remise ", ün tutęt " une fourchette", $\delta$ tuto " un couteau ", tüs " (une, la) cruche", patit piy " (une, la) petite fille ", wi pu fe pipi ma " oui, pour me faire faire

(1) Je prends cette occasion pour étendre la petite publicité déjà acquise à la formule d'un Ecolier candidat au C. E. P. : "Quand il y a plusieurs chevals on dit chevau, et quand il n'y a qu'un chevau on dit cheval., 
pipi », ni pa ni to " ni froid ni chaud ", ma ti qö $t \bar{z} z$ " moi aussi je veux quelque chose 1 .

33 - 6. "dans le " employé au lieu de " dans (1) ).

$35+9$. ev pa ta papa tupit ta do la li "lève-toi, sors, papa tout de suite, sors du lit" (voir pour Fr. p. 234 ; ici plus tardif).

$35+16$. Emploie le futur simple ou périphrastique; une phrase notée donne en orthographe française : " quand il viendra le train, (je) vais le jeter".

36. fo la epüte "il faut l'éplucher " et vö tü $l$ epüte "veux-tu l'éplucher ».

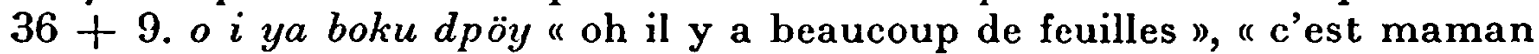
qu'on nous lave '.

$36+12$. (Pronom complément; relatif; conjonction; interrogation.)

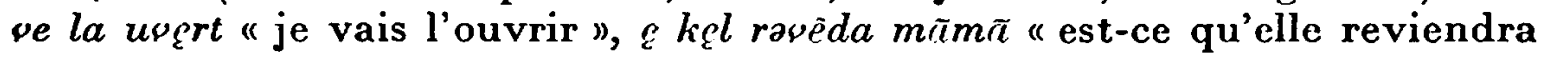
maman ».

sa fe dï bi kñ õ la føm " ga fait du bruit quand on la ferme ".

u ti sō le müzikè ti fö la müzik « où sont les musiciens qui font de la musique?» $36+20$. Emploie souvent "avant-demain" pour " maintenant, aujourd'hui ".

$36+24$. a la otr " à l'autre".

$363 / 4$ environ. N'emploie pas $j c, t u$, sauf "tu veux ", mais emploie " c'est moi, c'est toi " et " me "; te pa ma möt " c'est toi qui me monteras".

Noté une fois "elle " en parlant de sa mère à sa sœur " (je) veux compter seulement les bleus et les rouges".

37 - 4. tii pö matĩ " tu le peux le matin".

swïla il a de tavö " celui-là il a des cheveux ".

"c'est moi qui la l'ôte ".

" elle veut pas".

37 + 3. Au lieu du précédent " le bébé à toi " dit "celui à moi ", puis " le mien ", puis " mon bébé, il bave".

Le même jour demande de quelle couleur est un objet.

$37+5$. " tu veux bien lui render? ("rendre »).

$37+10$. " c'est cous" (= "cousu ").

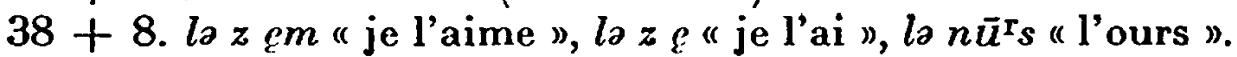

$38+17$. Dialogue entre elle et moi, devant trois poupées. J'en ai quatre [ca ne fait pas 4]. Un, deux, sept; j'en ai sept [non]. Un, huit, quatre [il y en a trois]. C'est celui-là trois; un, deux, trois [en désignant la poupée destinée à être no 3 ].

$38+23$. fo ot $i$ us $l \bar{t} t$ " il faut aussi ouvrir l'autre ».

39. te le bebe a ma "c'est mon bébé ».

39 + 3. sor ma da mo li "sors-moi de mon lit".

$39+4$. " il fallait me le jeter pour lui donner quand j'ai [= j'aurais eu $]$

fini de..."

$39+10$. vyē ma done mõ bibō " viens me donner mon biberon ".

$39+17$. pale tom tati "(tu) parlais comme Francis".

" quand je $\mathrm{l}(\mathrm{e})$ dirai à maman ".

" aujourd'hui ' est ogigi, puis ovodi.

$39+18$. lo pti peñ də pa pu mə pafe " ton petit peigne [le petit peigne de toi] pour me coiffer ".

40. "il fallait m'appeler " [pourquoi?] " parce que pour le défaire ".

(1) Langages successifs, p. 120-121; même endroit, sur a avec sans 》. 
$40+6$. se a ki « c'est à qui?? ».

$40+9$. " prends celle eau-là ".

$40+13$. vö tï pas ó pate " (je) veux (que) tu fasses un pâté 》 (subjonctil).

41. sür ma epôl puis sür mö epọl " sur mon épaule ".

42. [Avec déformation phonétique volontaire.] ४ö ma nune "je veux me coucher ".

$42+12$. ōn a zate " on a jeté ".

43 - 7. ek tü̈̈radi " qu'est-ce que tu lui as dit?"

ape zə tōb "après, je tombe ".

$43+12$. "quand je mettrai(s) [= si je mettais] le bracelet que Laurence m'a fait, peut-être il me enlèvera [= il me l'enlèverait].

$451 / 2$. il faut me couverte [= couvrir]; je ne peux pas me assise [= assoir].

4 ans 2 mois. [Taisez-vous les enfants.] " on nous tait", " ousqu'elle est maman et Laurence » et " maman et Laurence où qu'(ils) sont ».

4 ans 4 mois. la önyem "la première ".

Dit souvent des dates telles que " quand j'avais trois ans et demi et Laurence quatre ans et demi".

Quelques jours après, on a parlé de " la deuxième "; elle dit : mwa la ózyęm.

4 ans 6 mois. "[quand mon lit était fermé] tu me sortirais [= sortais], tu me descendais ".

[De 4 ans $1 / 2$ à 5 ans le phonétique adulte finit de s'établir. A 4 ans 7 mois, commence da apprendre à lire.]

4 ans 7 mois $1 / 2$. il faut que je l'ave [aie].

4 ans 10 mois. [Ne sait pas comment exprimer "continuer "] :

a vous la commencerez encore plus? vous la commencerez plus, plus plus?

"sans m'en aperçoir " [apercevoir].

*hacun auront un plat ".

" tu voulais pas que grand-mère le save [sache]".

4 ans 11 mois. "moi il faut que je soive [sois] là "; "voilà ce qu'il faut pour que ce soit très bien ».

4 ans 11 mois $1 / 2$. "quand ils avent [ont] ", mais après quelques minutes : ails ont".

5 ans. "quan(d) on partra (" partira ")».

i(ls) croivent (croient).

5 ans 15 jours. " je tiendais " ( (" tenais »).

5 ans 2 mois. " maman et Laurence est là ".

5 ans 3 mois. " est-ce qu'elle l'adore ou le désore " (= "déteste ").

" lettres écrises" (= "écrites").

"j'attends patieusement" (= " patiemment ").

5 ans 4 mois. "avant que tu me l'aves dit".

5 ans 11 mois. "papa et moi l'ont fini".

6 ans. " je suis couru" (= "j'ai couru ") " tu retniras " (" tu retiendras $).$ [Pour ils et elles. même référence que ci-dessus]

7 ans 6 mois. "le vois" pour " je le vois "; "veux le faire " pour " je veux le faire ".

7 ans 7 mois. "la carapace, c'est la peau dure de les bêtes".

7 ans 9 mois. quan(d) on s'est rejoigni " quand nous nous sommes rejoints *.

De 8 à 13 ans : seulement 32 observations, dont 7 pour ils-elles. 
J. 19 1/2. " mal au pied".

21. pati lo "il n'y a pas d'eau " (l'eau).

mane o pwa " aller me promener au bois».

akko pom " je voudrais une autre pomme".

Désignations de sa personne

22. Adjectif pepe miñõ "bébé mignon".

Adverbe de quantité ku apr " beaucoup d'arbres 》.

Le vocabulaire relevé à cette date permet d'établir une proportion des différentes parties du discours, d'après le classement en langage adulte. Le tableau résultant n'a qu'une valeur très douteuse pour ce qui est des proportions; d'une part un grand nombre des mots ne s ont pas encore différenciés dans le langage de l'enfant; d'autre part nous ne disposons pas de tableaux des proportions dans la langue adulte, soit dans le dictionnaire, soit dans l'usage moyen du milieu parisien qui est celui de l'entourage des sujets observés. On doit s'attendre, étant donné le caractère de la conversation des parents avec les enfants, à une certaine exagération du nombre des substantifs.

Substantifs . . . . . . . 213 Articles et pronoms . . . . . 1

Noms propres .. . . . . . 24 Adverbes. . . . . . . . 10

Verbes.......... 48 Prépositions ........ 0

Adjectifs . . . . . . 15 Interjections et analogues . . 20

D'autre part, il a été procédé à un classement sommaire par catégories de sens qui a donné les résultats suivants, une partie des verbes étant répartis avec les substantifs :

Personnes . . . . . . . . . 7 Ville et campagne. . . . . . 21

Personnes, individus et lieux . . 22 Animaux. . . . . . . . . . 12

Corps . . . . . . . . 29 Actions non réparties. . . . 24

Alimentation. . . . . . 45 Qualités ........ 9

Habillement . . . . . . 23 Expressions de relations entre

Hygiène . . . . . . . . . 22 les personnes (démonstratifs,

Jouets. . . . . . . . . . . 32 expressions de politesse, etc.) . 31

Habitation. . . . . . . 43

Les différences entre le total indiqué page 230 et les totaux de ces tableaux proviennent d'un petit nombre de répétitions ou d'omissions dans la répartition.

$221 / 2$. la pom "de la pomme".

bye "voilà, je l'ai fait".

mone ti pö " me promener un petit peu".

avęk papa "avec papa .

bęl ti tapr " beau petit arbre ".

$22+17$. A décomposé "corbeille à papier " de sorte qu'il la dénomme suivant ce qu'il y met : apap€y a baló, a bato "corbeille à bâton, à bateau ". $22+22$. tu ta lö (répété par jeu) " tout à l'heure ". 
$22+23$. ako la lętr " une autre lettre ".

tase la papye "le papier est déchiré [cassé]".

akko manan "encore de la banane".

ku $m i$ "beaucoup de fourmis".

la tam "la dame " (mot d'une syllabe; mais pasyö " monsieur ").

$22+24$. lep o pul ("porter ") l'herbe aux poules.

$22+25$. la lętr (avec $r$ sourd) " la lettre".

$22+29$. la pell.

Emploi de "autre ".

pati pato "petit bateau".

[A 23. On note des progrès rapides du langage en général.]

$23+1$. lo tüp "le tube".

Yeom "Jérôme ".

$23+2$. $t^{\mathrm{r}_{w}}$ a la pom équivaut à " $j$ 'ai un morceau de pomme dans chaque main " [pluralité].

$23+4$. S'amuse à dire ö tö e trwa " un deux et trois».

us la bwat "ouvre la boîte ".

ole pale " où est-il le balai?" ".

$23+5$. $i p^{2} l \ddot{o}$ " il pleut ».

[Noté par sa mère.[ $\mathrm{i}(\mathrm{l})$ do(rt) papa le li(t) "papa dort dans le lit ".

$23+6$. Zerōm "Jérôme".

$23+15$. D'une manière générale il n'interroge pas. On peut se demander s'il n'emploie pas, au lieu de questions, des propositions d'essai; ainsi prenant une pierre ponce il dit pom pe "pomme de pin "; je dis "non, ce n'est pas une pomme de pin "; alors il énonce pati kayu " petit caillou ".

mot'e gräme a mi grüpe " je montre à grand-mère comment grand-père me l'a mis.

promne avęk mãmĩ tu ta lö "me promener avec maman tout à l'heure". pappa i to " papa (il) dort ».

dï pẽ " du pain ".

$23+18$. "son papa".

$23+24$. [Témoignage de sa mère.] Papa et Maman et Jérôme.

" autre une dame" (en image).

"Jérôme tout fini l'œuf ".

Noté par moi : yę " hier".

24 - 2. mim $\pi$ e e papa " maman et papa ".

fo dodo avęk papa mimm $\pi$ " maman est couchée avec papa 》.

la balass " la balance ".

$i$ blö la bebe " $i l$ pleure le bébé ".

sür grĩ me "sur grand-mère ".

dade la liv $\mathbf{r}$ "....regarder le livre" ".

dade mi lio "...regarder mon livre".

se bả la la bswat "la boîte n'est pas là (c'est pas là...) ".

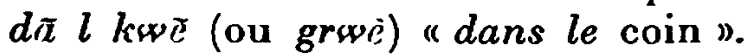

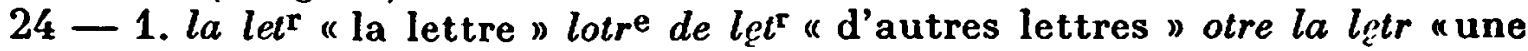
autre lettre $"$.

$24+2$. "les deux les pêches " = "deux pêches .

$24+12$. desăd avgk grämęr " je suis descendu avec grand-mère " (passé non exprimé). 
grāpe a mi ã o la bsvat "grand-père a mis en haut la boîte " (passé exprimé). pakã tro sal "...parce que c'est très sale ".

[Sa mère témoigne qu'il entretient des fictions; quand une personne téléphone, il dit que c'est une autre, etc., sciemment.]

$25-6$. [Notes de sa grand-mère.] dans la fenêtre; Jérôme prend la bobine à Viroflay de grand-mère; à côté de le pot; Jérôme ramassé ma-broche, parce que Jérôme a fait tomber ma-broche de Christiane " (ma agglutiné).

Noter l'emploi de "parce que ".

"deux le bouton" = les deux boutons.

" c'est Christiane donné la petite auto à Jérôme (absence de "qui ").

$25+2$. Phrases. Il regarde son chapeau de Jérôme sur la glace. Papa met le chapeau de Jérôme sur la tête. Papa donne le chapeau. Papa met le chapeau de Jérôme sur la tête sur les cheveux comme Jérôme.

" comme " est très employé.

"deux la poule" (" deux poules").

"le crayon pour grand-père; l'autre le crayon; c'est le stylo pour grandpère pour écrire dans le papier" ".

$25+12$. [tu as bien dormi?] d $\pi^{\mathbf{r}}$ la $l i$ " dans le lit " (évite de répondre oui).

[3 œufs sur une image] les deux œufs-les deux l'œuf pour papa et pour maman et pour Jérôme. (Queue de phrase fréquente; d'autres fois il dit : comme papa et comme maman et comme Jérôme.)

$25+13$. " est arrangé »(= " il est arrangé »).

$25+14$. "le dé pour coudre ".

Raconte : "a fait boum " [c'est tombé]. Répète : " a fait boum " (au lieu de dire oui).

$25+15$. "(i)l a bien dormi » (= "j'ai bien dormi »).

" $a$ bien dormi les vaches".

" $t u$ dis au revoir à grand-père" (= je dis...).

$25+17$. [Lettre de L.] A dit, en ponctuant ce qu'il voyait : " maman boit " puis " maman a bu \#- " je voudrais du sable ".

26 - 4. [Lettre de L.] " moi, j'entends Marie ".

26 - 3. [Témoignage de son père.] " moi je vois pas " [id.] « je le mange ». 26. "je porte à maman ".

$26+2$. "je veux pas ", " je voudrais le sucre ».

$26+3$. J'évite de lui parler à la $3^{e}$ personne; je lui dis : "c'est pour toi ", "c'est pour moi ". Le soir, dit (sauf erreur de détail) " c'est pour Jérôme, à toi ».

26 + 4. "après biscotte, donnera(s) une pêche " (premier emploi noté du futur).

"il grimpe "(= " je grimpe »).

On lui dit : "il faut attendre parce que c'est chaud "; répond : "attendre, c'est chaud ".

26 + 5. ręn grã pęr "j'aide grand-père ", et tout de suite après eqde Mari "j'aide Marie ".

$26+9$. "c'est Jérôme qui la mange ".

S'adressant à moi, demande un râteau que je tiens en disant " c'est pour toi " (= c'est pour moi); je réponds : " non, c'est pour moi "; ne dit plus rien; [je lui demande " qu'est-ce que c'est? " réponse "balai »; voir ci-dessus p. 226]. Avee ses parents, continue à dire " pour Jérôme". 
$26+10$. «il a pleuré »(en parlant de lui).

$26+16$. Pour parler de lui, à côté de "Jérôme » emploie quelque chose qui semble être une abréviation Om: om øö gato " je veux du gâteau " (une confusion partielle avec " on " est peut-être à envisager?).

Vient m'accompagner au jardin en disant "Aller travailler " - "Tu veux travailler?" - "Travailler avec moi " (= avec toi). L'inversion des personnes continue.

$26+19$. Emploi de "parce que ".

Il a deux carottes, j'en ajoute une; il dit " une autre carotte "; je dis : trois; pas de réponse.

râd $k \ddot{o}$ " (il a une) grande queue " (un coquelicot).

S'amuse à compter : un, deux, trois, quatre, six, huit, neuf et dix.

$26+21$. ["Qu'est-ce que c'est? "] " couteau " [" un couteau? 》] " un couteau " ["à qui est-il? "] " de Jérôme ».

$26+22$. En promenade avec ses grands-parents dit plusieurs fois " tous les deux", puis "tous les trois", puis alterne les deux.

27 - 4. (Lettre de sa mère.) A dit : " j'ai prendu le livre " (précédemment disait : "Jérôme a pris »; possède depuis longtemps le mot " descendu ".

"il rigole".

$27+5$. "c'est cuisu " (= c'est cuit). Cette forme n'a pas persisté.

27 + 16. [Lettre de sa mère.] A dit précédemment "l'autre l'éponge "; maintenant "l'éponge et les autres ponges"; de même "l'assiette et la petite siette "; "la grande moire" (= armoire).

On lui dit " envoie un baiser "; répond " je sais pas".

Phrase sans " je " - "Jérôme aide maman à étendre le linge ".

J. L. : $19+8$. pü "il n'y en a plus" (voir p. 222).

$211 / 2$. dö " deux, une paire, plusieurs".

$21+22$. bii " j'ai bu ".

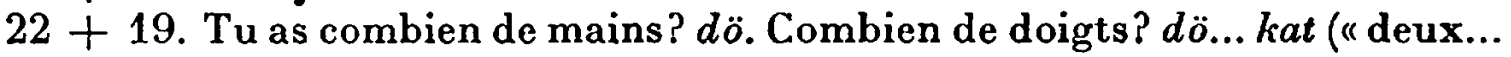
quatre $)$.

$a \ddot{o}$ " tout à l'heure ".

$b \ddot{i}$ " j'ai bu tout à l'heure ".

ta te " je veux aller par terre".

23 - 4. [Combien de bateaux?] dö-tat (2-4; en réalité 3).

pato dã "les bateaux sont dans la poche".

$24+1$. o " encore ".

$24+4$ aye pa " ça n'y est pas".

$24+11 \tilde{o}$ pato " un bateau " s'opposant à dö pato "deux bateaux " n'est pas nettement article.

$24+16$. pati vato " petit bateau ".

sãd ̈̈ « je suis descendu (descendre?) ".

$24+15$. e mõte " je suis monté ".

$24+22 . \pi p e \quad m \tilde{a} \tilde{z}$ " grand-père mange ".

$25+4$. Emploie souvent " aussi ".

$\tilde{z} \tilde{a}$ vi osi " Jean-Louis aussi ".

$25+23$. la dö apẽ « là, deux lapins ".

bubin trswa " trois bobines " (compte exact par hasard?).

Distingue des couleurs, sur une robe, des lapins, des épingles à linge. 
" mange une pomme, grand-mère ».

$25+24$. "(je) veux un yaourt; pas deux; un (insisté) yaourt; un yaourt; ah voilà yaourt ".

" de l'eau ".

ave $a$ tęt "je me suis lavé la tête ".

awa dö bõbõ sola " je vais avoir 2 bonbons de chocolat ".

$25+26$. $d \tilde{a} l i$ " dans le lit " (préposition, pas d'article).

26 - 3. Raconte à son père son voyage qui a eu lieu neuf jours avant,

à peu près ainsi : " prené train hier, a vu Vienne, loin Seine ".

"dans hangar").

" descender " (" descendre? " On lui a souvent dit "descendez ».)

26 - 2. "des pommes".

$26-1$. [Son père : lu es mon mignon?] "ton mignon ".

[A qui est ca?] "c'est à toi ». [Qui c'est toi] "c'est à Jean-Louis ».

Je lui dis : "Tais-toi "; il répète " tais-toi ".

Spontané : "bonjour grand-père; mange (= il mange) une poire, grandpère ".

" une auto passe là... partie une auto".

" monter sur la table "; pour maman".

ün taot "une carotte".

Il joue avec 4 cuillers en disant dö tssa wit tat $2,3,8,4$.

26 mois. Le vocabulaire relevé à cette date a fourni les chiffres suivants (se reporter aux observations de la p. 243) :

Substantifs : 294; noms propres 42.

Verbes : 68 .

Adjectifs : 27.

Articles et pronoms : 12 .

Adverbes : 18.

Prépositions : 8 .

Interjections et analogues : 15 .

Nombres : 10.

La répartition d'après les sens n'a pas été faite. Toutes choses égales d'ailleurs, c'est-à-dire sans penser que J.-L. aurait fourni les mêmes chiffres que J. à l'âge où celui-ci a été sondé, on peut comparer grosso modo les deux tableaux, en observant d'une part les totaux, qui sont symptomatiques de la croissance, et aussi les progressions inégales des différentes catégories.

26. fora plï " je ne le ferai plus » asye twva la "assieds-toi là ».

A son père : "ça, lait pour Yves, avec Jean-Louis" (= tu tiens la boîte pour faire du lait pour Yves; je veux le faire avec toi ").

[Tu as mangé ta banane?] mã̃e "je l'ai mangée " [évite de dire oui]. [donne-le moi] "donne ", puis "donne-le-moi".

[qu'est-ce que tu as dit à papa?] " manger ton café " (l'emploi correct du possessif n'est pas spontané; on lui avait dit : dis à papa : viens boire ton café, ou analogue).

$26+1$. "pourrais avoir ca? " (interrogation de politesse).

$26+2$. "va (= je vais) porter à maman celle-là ".

Identifie correctement des couleurs sur une couverture de livre.

$26+3$. [Lettre de sa mère] : présent "Renée arrive "; passé " $\mathrm{X}$ a fait *. 
F.. R. 18. "pas là, papoune (grand-père). [D'autre part fait non de la tête.]

19. " beau rose " (" belle rose ").

"a pas là »= "n'est pas là, il n'y a pas là \#.

" encore ".

A 20. On note : grand progrès.

"bon sucre".

"voir ta fille".

21. "beaucoup de l'eau".

"assez!".

24. Un mot maka a le sens "encore "; mais il y a aussi äkonün "encore une $)$.

"gros monsieur ", "bien mignon papa".

" mets les chaussures" (article).

" les rentré " interprété " je les ai rentrées" (pronom).

" maman, tu veux mettre chaussures" (= " je veux ").

\section{Observations finales}

Dans le plan d'étude proposé ci-dessus il a été fait effort pour serrer d'assez près la réalité, et il en résulte, de manière non inattendue, que si on constate bien dans la période d'acquisition des aspects successifs du langage il est très difficile de délimiter les stades et de leur donner des dénominations adéquates.

Les datations par les débuts se révèlent fallacieuses. Voici en effet ce que donnent à peu près les tableaux d'exemples de l'exposé cidessus, en ne prenant que les dates de début des périodes.

\begin{tabular}{|c|c|c|c|c|c|c|}
\hline & Fr. & L. & Chr. & $\mathbf{J}$ & J.-L. & Fr. R. \\
\hline Roucoulement. . . & $21 / 2$ & $11 / 4$ & $11 / 2$ & 2 & 3 & $?$ \\
\hline Gazouillis . . . . . & $51 / 2$ & $21 / 2$ & $21 / 2$ & $21 / 4$ & 5 & ? \\
\hline $\begin{array}{l}\text { Premiers éléments de } \\
\text { communication. }\end{array}$ & 9 & $92 / 3$ & $81 / 2$ & 10 & 10 & $?$ \\
\hline $\begin{array}{l}\text { cléments de commu- } \\
\text { nication multipliés. } \\
\text { Apparition des as- }\end{array}$ & 16 & 15 & $161 / 2$ & $(141 / 2 ?) 191 / 4$ & 18 & $?$ \\
\hline $\begin{array}{l}\text { semblages. } \cdot \text { d'élé- } \\
\text { Apparition } \\
\text { ments du langage }\end{array}$ & 19 & $(143 / 4) 16$ & 21 & $191 / 4$ & $192 / 3$ & 12 \\
\hline adulte. . . . . & 21 & $191 / 2$ & 22 & $191 / 2$ & $191 / 2$ & 18 \\
\hline
\end{tabular}

Pour certains des enfants, il arrive que le début d'un stade supérieur coïncide ou presque avec celui du stade précédent. Pourtant ils ont tous présenté - avec quelques différences d'âge - la progression que représentent grosso modo les dénominations données. Ces dénominations utiles ne représentent qu'une partie des éléments de croissance, qu'on doit considérer en bloc : ainsi le progrès d'exactitude dans la prononciation va de pair avec l'allongement des ássemblages et ensuite avec eelui des phrases proprement dites. 
Il faudrait pouvoir apprécier par des coupes rapprochées et aussi complètes que possible les proportions respectives des éléments, spécialement celle des éléments isolés par rapport à celle des éléments en assemblage (en distinguant la nature et la longueur de ces assemblages), et de même pour les proportions des éléments de langage adulte.

Ces proportions ne devraient pas être obtenues en comptant chaque trait observé pour une unité, mais en tenant compte du nombre d'apparitions dans un espace de temps donné : les fréquences d'emploi sont un élément essentiel d'appréciation.

Si on pouvait opérer ainsi, on définirait sans doute des périodes caractéristiques du maximum de fonctionnement de chaque système provisoire successif. On apercevrait par contraste quel est l'aspect des périodes de passage, avec les chevauchements ou endentements irréguliers causés par les emplois avant-coureurs ou, inversement, persistants de manière anormale.

Espérons que nos successeurs pourront faire de pareils travaux.

En attendant, quelles conclusions tirer de ce que nous avons pu touf de même entrevoir dans notre recherche?

Une première conclusion est négative : le "stade des quelques mots " dont on a commencé à parler vers 1920, et dont j'ai fait état moi-même, ne paraît pas à retenir - surtout si on s'abstient de parler de " mots " pour la période soi-disant caractérisée par ce stade.

Il est peut-être permis d'insister sur des points positifs.

Dans la majeure partie, semble-t-il, de la période où dominent les éléments isolés, il existe une répartition des emplois entre des éléments non parlés, cris et grognements, qui ont généralement un rôle affectif, et des éléments parlés (mots du langage adulte très raccourcis et à phonèmes souvent mal ou non articulés) qui servent principalement à la conversation. Si on considère les choses de cette façon, on doit conclure que l'emploi abondant d'éléments de communication non affectifs commence très tôt.

De manière analogue, si on veut bien tenir compte que des éléments de communication non parlés, surtout certains gestes et certains grognements, peuvent entrer comme partie composante dans des assemblages affectifs ou non, on admettra que les assemblages (équivalant à des phrases à plus d'un mot) sont plus variés et aussi plus précoces qu'on ne le croit généralement.

Les recherches ultérieures confirmeront-elles ces points de vue? Quoi qu'il en soit, il faut espérer que des observations variées et massives, dégagées d'un certain nombre d'idées préconçues anciennes et faites avec l'aide de procédés modernes, permettront de faire progresser une étude nécessaire. 